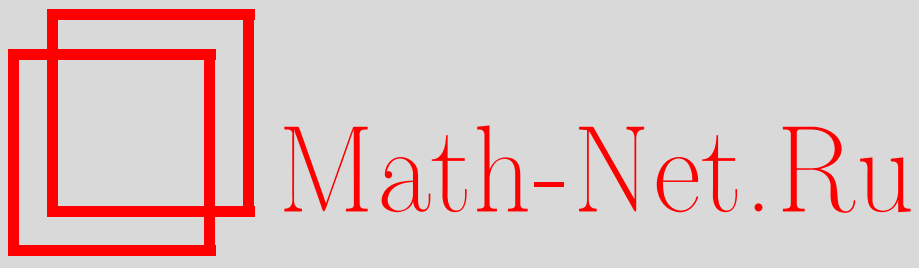

А. С. Сорокин, Структурные формулы некоторых классов аналитических функций в конечносвязной области, Матем. сб., 1997, том 188, номер 12, 107-134

DOI: https://doi.org/10.4213/sm281

Использование Общероссийского математического портала Math-Net.Ru подразумевает, что вы прочитали и согласны с пользовательским соглашением http: //www. mathnet.ru/rus/agreement

Параметры загрузки:

IP: 18.207 .199 .55

26 апреля 2023 г., $16: 39: 10$ 
УДК 517.54

\author{
А. С. Сорокин
}

\title{
Структурные формулы некоторых классов аналитических функций в конечносвязной области
}

\begin{abstract}
Дается распространение центральных теорем метода структурных формул для некоторых классов аналитических функций, полученњых ранее для круга и кольца, на аналитические функции в конечносвязной круговой области.

Библиографоия: 10 названий.
\end{abstract}

В настоящей работе центральные теоремы метода структурных формул для некоторых классов аналитических функций, полученные ранее для круга и кольца, распространены на аналитические функции в конечносвязной круговой области.

Вывод формул основан на уже известных интегральных представлениях аналитических функций в конечносвязной круговой области (см. [1]-[3]).

Эти интегральные представления получены методом функциональных уравнений, основанным на разделении особенностей функции преобразования и на сведении задачи ее определения к системе функциональных уравнений, которые затем решаются методом последовательных подстановок.

В [1] было показано, как надлежашим изменением функциональных уравнений и методом последовательных подстановок достигается решение задачи для областей с любьм расположением граничных кругов.

Указанные в теоремах 1.1-3.2 формулы, обобщающие структурные формулы на случай конечносвязных круговых областей, представляют собой развитие и усиление результатов Зморовича [4], [5], Паатеро [6], [7].

Известная структурная формула класса выпуклых, однолистных функций, регулярных в круге, была обобщена в 1953 году Зморовичем на случай кругового кольца [4].

В 1961 году Максимов [8] получил дальнейшее обобщение этой формулы на случай многосвязной круговой области. Решение дано в конструктивной форме, однако не для всех областей, а только для тех, которые удовлетворяют определенньм условиям, обеспечивающим сходимость рядов, участвующих в образовании структурной формулы. Эти условия, говоря неформально, будут выполнены, когда радиусы внутренних окружностей конечносвязной круговой области достаточно малы, а радиус внешней окружности, охватываюшей остальные, достаточно велик.

Из теорем 5.1, 5.2, содержащих структурные формулы для конечносвязных круговых областей с любым расположением граничных кругов, легко выводятся результаты Максимова [8]. 


\section{$\S 1$. Структурная формула для аналитических функций в классе $\mathscr{B}\left(R_{j}, a_{j}, j=0, \ldots, n\right)$}

Пусть вещественные числа $R_{k}>0$ и комплексные числа $a_{k}\left(a_{0}=a_{n}=0\right)$, $k=0,1, \ldots, n$, таковы, что выполнены неравенства

$$
\left|a_{k}\right|<R_{0}-R_{k}, \quad\left|a_{k}-a_{j}\right|>R_{k}+R_{j}, \quad k \neq j, \quad k, j=1, \ldots, n .
$$

Будем рассматривать область $\mathscr{K}\left(R_{j}, a_{j}, j=0, \ldots, n\right)$ в плоскости $z$, представляющую собой пересечение круга $\mathscr{D}_{0}:|z|<R_{0}$ с внешностью кругов $\mathscr{D}_{k}$ : $\left|z-a_{k}\right| \geqslant R_{k}, k=1, \ldots, n$, где $a_{k}$ и $R_{k}$ удовлетворяют условиям (1.1), и называть ее $(n+1)$-связной круговой областью.

Назовем функиией класса $\mathrm{VB}[a, b]$ каждую однозначную на сегменте $[a, b]$ вещественную функцию $\mu(\vartheta)$ с ограниченным изменением на этом сегменте, нормированную условием $\mu(a+0)=\mu(a)=0$.

Далее, назовем функиией класса $\mathscr{B}\left(R_{j}, a_{j}, j=0, \ldots, n\right)$ каждую однозначную аналитическую в круговой конечносвязной области $\mathscr{K}\left(R_{j}, a_{j}, j=0, \ldots, n\right)$ функцию $f(z)$, удовлетворяющую условию

$$
\begin{gathered}
\max _{0 \leqslant k \leqslant n} \int_{-\pi}^{\pi}\left|\operatorname{Re} f\left(a_{k}+\rho \exp (i \vartheta)\right)\right| d \vartheta<L(f), \\
R_{k}<\rho<R_{k}^{0}=\min _{\substack{1 \leqslant m \leqslant n \\
m \neq k}}\left\{\left|a_{m}-a_{k}\right|-R_{m}\right\}, \quad k=1, \ldots, n, \\
R_{0}>\rho>R_{0}^{0}=\max _{1 \leqslant m \leqslant n-1}\left\{R_{m}+\left|a_{m}\right|\right\},
\end{gathered}
$$

где $L(f)$ - некоторая положительная постоянная, зависящая, вообще говоря, от выбора функции $f(z)$.

Теорема 1.1. Пусть $\widetilde{\mu}_{k}(\vartheta)=\mu_{k}\left(a_{k}+R_{k} \exp (i \vartheta)\right), k=0,1, \ldots, n,-\oint у н к u и и ~$ класса $\mathrm{VB}[-\pi, \pi]$, удовлетворяюшие соотношениям

$\mathscr{A}_{m}=\frac{1}{2 \pi} \int_{-\pi}^{\pi} d \widetilde{\mu}_{m}(\vartheta)=\sum_{\substack{k=0 \\ k \neq m}}^{n} \frac{\delta_{k}}{2 \pi} \int_{-\pi}^{\pi} S_{k}\left(a_{k}+R_{k} \exp (i \vartheta)\right) d \widetilde{\mu}_{k}(\vartheta), \quad m=1, \ldots, n$,

гдe

$$
\begin{aligned}
& S_{m}(\zeta)=1+\frac{1}{2} \sum_{\nu=0}^{\infty} \sum_{k_{1} \neq k_{2}}^{k_{2 \nu} \neq k} \sum_{\substack{k=0 \\
k \neq m}}^{n}\left(\left.\frac{\zeta+\overline{L_{m}} E_{2 \nu} L_{k}(z)}{\zeta-\overline{L_{m}} E_{2 \nu} L_{k}(z)}\right|_{b_{k}} ^{a_{m}}-\left.\frac{\zeta+\overline{E_{2 \nu} L_{k}}(z)}{\zeta-\overline{E_{2 \nu} L_{k}}(z)}\right|_{\overline{b_{k}}} ^{\overline{a_{m}}}\right) \\
& b_{0}=0, b_{m}=\infty, m=1, \ldots, n, \delta_{k}-\text { символ Кронекера }[1] \text {. } \\
& \text { При әтом } \\
& \begin{array}{c}
E_{2 \nu}(z)=L_{k_{1}} \overline{L_{k_{2}}} \cdots L_{k_{2 \nu-1}} \overline{L_{k_{2 \nu}}}(z), \quad \nu=1,2, \ldots, \\
E_{0}(z) \equiv z
\end{array}
\end{aligned}
$$


Кроме того,

$$
L_{k}(\zeta)=\overline{a_{k}}+\frac{R_{k}^{2}}{\zeta-a_{k}}
$$

Далее, пусть

$$
\begin{aligned}
\mathscr{A}_{0}+i \mathscr{D} & =\frac{1}{2 \pi i} \int_{|z|=\rho} \frac{f(z)}{z} d z, \\
R_{0}>\rho>R_{0}^{0} & =\max _{1 \leqslant m \leqslant n-1}\left\{R_{m}+\left|a_{m}\right|\right\} .
\end{aligned}
$$

Тогда структурная формула

$$
f(z)=\sum_{k=0}^{n} \frac{\delta_{k}}{2 \pi} \int_{-\pi}^{\pi} H_{k}\left(a_{k}+R_{k} \exp (i \vartheta), z\right) d \widetilde{\mu}_{k}(\vartheta)+\mathscr{A}_{0}+i \mathscr{D}
$$

əдe

$$
H_{k}(\zeta, z)=\left.\sum_{\nu=0}^{\infty} \sum_{k_{1} \neq k_{2}}^{k_{2 \nu} \neq m} \sum_{m=0}^{n} \frac{\zeta+\overline{L_{k}} E_{2 \nu} L_{m}(z)}{\zeta-\overline{L_{k}} E_{2 \nu} L_{m}(z)}\right|_{b_{m}} ^{z}
$$

является необходимым и достаточным условием принадлехсности функции $f(z)$ классу $\mathscr{B}\left(R_{j}, a_{j}, j=0, \ldots, n\right)$.

ДоказАТЕЛьСтво. Достаточность. Из формулы (1.11) следует

$$
\begin{aligned}
& \operatorname{Re}\left(f\left(a_{m}+R_{m} \exp (i \vartheta)\right)\right) \\
& =\mathscr{A}_{0}+\sum_{k=0}^{n} \frac{\delta_{k}}{2 \pi} \int_{-\pi}^{\pi} \operatorname{Re}\left(H_{k}\left(a_{k}+R_{k} \exp (i \vartheta), a_{m}+R_{m} \exp (i \varphi)\right)\right) d \widetilde{\mu}_{k}(\varphi),
\end{aligned}
$$

$m=0,1, \ldots, n$. Очевидно, что каждую функцию $\widetilde{\mu}_{k}(\varphi), \quad k=0,1, \ldots, n$, можно представить в виде

$$
\widetilde{\mu}_{m}(\varphi)=\sigma_{m}(\varphi)-\tau_{m}(\varphi), \quad m=0,1, \ldots, n
$$

где $\sigma_{m}(\varphi)$ и $\tau_{m}(\varphi)$ - неубываюшие на $[-\pi, \pi]$ функции с ограниченньм изменением. Тогда, учитывая, что $\operatorname{Re} H_{k}(\zeta, z)>0, \zeta \in \mathscr{C}_{k}(\zeta)\left(\mathscr{C}_{k}(\zeta):\left|\zeta-a_{k}\right|=R_{k}\right.$, $k=0,1, \ldots, n), z \in \mathscr{K}\left(R_{j}, a_{j}, j=0, \ldots, n\right)$, из (1.13) имеем

$\int_{-\pi}^{\pi}\left|\operatorname{Re} f\left(a_{k}+R_{k} \exp (i \vartheta)\right)\right| d \vartheta \leqslant \sum_{m=0}^{n}\left(\frac{1}{2 \pi} \int_{-\pi}^{\pi} d \sigma_{m}(\varphi)+\frac{1}{2 \pi} \int_{-\pi}^{\pi} d \tau_{m}(\varphi)\right)+\left|\mathscr{A}_{0}\right|$

$k=0,1, \ldots, n$. Отсюда следует, что функция $f(z)$ принадлежит классу $\mathscr{B}\left(R_{j}, a_{j}, j=0, \ldots, n\right)$.

Необходимость. Рассмотрим функцию

$$
H_{k}\left(\zeta, z ; r_{0}\right)=\left.\sum_{\nu=0}^{\infty} \sum_{k_{1} \neq k_{2}}^{k_{2 \nu} \neq m} \sum_{m=0}^{n} \frac{\zeta+\overline{\mathscr{L}_{k}} \mathscr{E}_{2 \nu} \mathscr{L}_{m}(z)}{\zeta-\overline{\mathscr{L}_{k}} \mathscr{E}_{2 \nu} \mathscr{L}_{m}(z)}\right|_{b_{m}} ^{z}
$$


где $0<r_{0}<1$,

$$
\mathscr{L}_{k}(z)=\overline{a_{k}}+\frac{\left(R_{k} r_{0}^{\delta_{k}}\right)^{2}}{z-a_{k}}, \quad \mathscr{E}_{2 \nu}(z)=\mathscr{L}_{k_{1}} \overline{\mathscr{L}_{k_{2}}} \cdots \overline{\mathscr{L}_{k_{2 \nu}}}(z)
$$

Ядерная функция $H_{k}\left(\zeta, z ; r_{0}\right)$ играет для круговой конечносвязной области $\mathscr{K}\left(R_{j} r_{0}^{\delta_{j}}, a_{j}, j=0, \ldots, n\right)$ ту же роль, что и функция $H_{k}(\zeta, z)$ для круговой конечносвязной области $\mathscr{K}\left(R_{j}, a_{j}, j=0, \ldots, n\right)$.

Очевидно, что

$$
\operatorname{Re} H_{k}\left(\zeta, z ; r_{0}\right) \rightarrow \operatorname{Re} H_{k}(\zeta, z) \text { при } r_{0} \rightarrow 1
$$

равномерно относительно $z$. Поскольку функция $f(z)$ регулярна в круговой конечносвязной области $\mathscr{K}\left(R_{j} r_{0}^{\delta_{j}}, a_{j}, j=0, \ldots, n\right)$, то ее можно представить в этой области формулой

$$
f(z)=\sum_{k=0}^{n} \frac{\delta_{k}}{2 \pi} \int_{-\pi}^{\pi} U_{k}\left(R_{k} r_{0}^{\delta_{k}}, \vartheta\right) H_{k}\left(a_{k}+R_{k} \exp (i \vartheta), z ; r_{0}\right) d \vartheta+\mathscr{A}_{0}+i \mathscr{D}
$$

где

$$
\begin{aligned}
U_{k}(r, \varphi) & =\operatorname{Re} f\left(a_{k}+r \exp (i \varphi)\right), \quad-\pi \leqslant \varphi \leqslant \pi, \\
R_{k}<r<R_{k}^{0} & =\min _{\substack{1 \leqslant m \leqslant n \\
m \neq k}}\left\{\left|a_{m}-a_{k}\right|-R_{m}\right\}, \quad k=1, \ldots, n, \\
R_{0}>r>R_{0}^{0} & =\max _{1 \leqslant m \leqslant n-1}\left\{R_{m}+\left|a_{m}\right|\right\}
\end{aligned}
$$

(см. [1]-[3]). Причем

$$
\begin{aligned}
\mathscr{A}_{m} & =\frac{1}{2 \pi} \int_{-\pi}^{\pi} U_{m}\left(R_{m} r_{0}^{\delta_{m}}, \varphi\right) d \varphi \\
& =\sum_{\substack{k=0 \\
k \neq m}}^{n} \frac{\delta_{k}}{2 \pi} \int_{-\pi}^{\pi} U_{k}\left(R_{k} r_{0}^{\delta_{k}}, \varphi\right) S_{k}\left(a_{k}+R_{k} \exp (i \varphi)\right) d \varphi, \quad m=1, \ldots, n .
\end{aligned}
$$

Кроме того,

$$
\begin{aligned}
\mathscr{A}_{0}+i \mathscr{D} & =\frac{1}{2 \pi i} \int_{|z|=r} \frac{f(z)}{z} d z, \\
R_{0}>r>R_{0}^{0} & =\max _{1 \leqslant m \leqslant n-1}\left\{R_{m}+\left|a_{m}\right|\right\} .
\end{aligned}
$$

Из формулы (1.16) получаем

$$
U(r, \vartheta)=\mathscr{A}_{0}+\sum_{k=0}^{n} \frac{\delta_{k}}{2 \pi} \int_{-\pi}^{\pi} U_{k}\left(R_{k} r_{0}^{\delta_{k}}, \varphi\right) \operatorname{Re} H_{k}\left(a_{k}+R_{k} r_{0}^{\delta_{k}} \exp (i \varphi), r \exp (i \vartheta) ; r_{0}\right) d \varphi
$$


Здесь $r \exp (i \vartheta) \in \mathscr{K}\left(R_{j} r_{0}^{\delta_{j}}, a_{j}, j=0, \ldots, n\right)$.

Пусть величина $r_{0}$ в формуле $(1.17)$ пробегает последовательность значений $r_{p}$, где $r_{p} \rightarrow 1$ при $p \rightarrow+\infty$, причем $0<r_{p}<1, p=1,2, \ldots$.

Положим

$$
U(\rho, \varphi)=U^{+}(\rho, \varphi)-U^{-}(\rho, \varphi)
$$

где

$$
\begin{aligned}
& U^{+}(\rho, \varphi)=U(\rho, \varphi) \quad \text { при } U(\rho, \varphi) \geqslant 0, \\
& U^{-}(\rho, \varphi)=-U(\rho, \varphi) \text { при } U(\rho, \varphi) \leqslant 0 .
\end{aligned}
$$

Тогда

$$
|U(\rho, \varphi)|=U^{+}(\rho, \varphi)+U^{-}(\rho, \varphi)
$$

и из условия

$$
\begin{gathered}
\max _{0 \leqslant k \leqslant n} \int_{-\pi}^{\pi}\left|U\left(\rho_{k}, \varphi\right)\right| d \varphi<L, \\
R_{k}<\rho_{k}<R_{k}^{0}=\min _{\substack{1 \leqslant m \leqslant n \\
m \neq k}}\left\{\left|a_{m}-a_{k}\right|-R_{m}\right\}, \quad k=1, \ldots, n, \\
R_{0}>\rho_{0}>R_{0}^{0}=\max _{1 \leqslant m \leqslant n-1}\left\{R_{m}+\left|a_{m}\right|\right\}
\end{gathered}
$$

имеем

$$
\begin{gathered}
\max _{0 \leqslant k \leqslant n} \int_{-\pi}^{\pi} U^{+}\left(\rho_{k}, \varphi\right) d \varphi<L, \quad \max _{0 \leqslant k \leqslant n} \int_{-\pi}^{\pi} U^{-}\left(\rho_{k}, \varphi\right) d \varphi<L \\
R_{k}<\rho_{k}<R_{k}^{0}=\min _{\substack{1 \leqslant m \leqslant n \\
m \neq k}}\left\{\left|a_{m}-a_{k}\right|-R_{m}\right\}, \quad k=1, \ldots, n \\
R_{0}>\rho_{0}>R_{0}^{0}=\max _{1 \leqslant m \leqslant n-1}\left\{R_{m}+\left|a_{m}\right|\right\}
\end{gathered}
$$

Пользуясь (1.19), формулу (1.17) можно представить в виде

$$
U(r, \vartheta)=\mathscr{A}_{0}+\sum_{k=0}^{n} \frac{\delta_{k}}{2 \pi} \int_{-\pi}^{\pi} \operatorname{Re} H_{k}\left(a_{k}+R_{k} r_{p}^{\delta_{k}} \exp (i \varphi), r \exp (i \vartheta) ; r_{p}\right) d \mu_{k}^{(p)}(\varphi)
$$

где

$$
\mu_{m}^{(p)}(\varphi)=\sigma_{m}^{(p)}(\varphi)-\tau_{m}^{(p)}(\varphi), \quad m=0,1, \ldots, n
$$

причем

$$
\begin{gathered}
\sigma_{m}^{(p)}(\varphi)=\int_{-\pi}^{\varphi} U_{m}^{+}\left(\rho_{j, p}, \psi\right) d \psi, \quad \tau_{m}^{(p)}(\varphi)=\int_{-\pi}^{\varphi} U_{m}^{-}\left(\rho_{j, p}, \psi\right) d \psi \\
\rho_{j, p}=R_{j} r_{p}^{\delta_{j}}
\end{gathered}
$$

Легко видеть, что функции $\sigma_{m}^{(p)}(\varphi), \tau_{m}^{(p)}(\varphi), m=0,1, \ldots, n,-$ неубывающие на сегменте $[-\pi, \pi]$. 
Кроме того,

$$
\mu_{m}^{(p)}(-\pi+0)=\mu_{m}^{(p)}(-\pi)=0, \quad m=0,1, \ldots, n,
$$

и

$$
\begin{aligned}
\mathscr{A}_{m} & =\frac{1}{2 \pi} \int_{-\pi}^{\pi} d \mu_{m}^{(p)}(\varphi)=\sum_{\substack{k=0 \\
k \neq m}}^{n} \frac{\delta_{k}}{2 \pi} \int_{-\pi}^{\pi} S_{k}\left(a_{k}+R_{k} \exp (i \varphi)\right) d \mu_{k}^{(p)}(\varphi) \\
& =\frac{1}{2 \pi} \int_{-\pi}^{\pi} U_{m}(\rho, \varphi) d \varphi=\sum_{\substack{k=0 \\
k \neq m}}^{n} \frac{\delta_{k}}{2 \pi} \int_{-\pi}^{\pi} U_{k}(\rho, \varphi) S_{k}\left(a_{k}+R_{k} \exp (i \varphi)\right) d \varphi
\end{aligned}
$$

$m=1, \ldots, n$, где

$$
\begin{aligned}
& R_{k}<\rho<R_{k}^{0}=\min _{\substack{1 \leqslant m \leqslant n \\
m \neq k}}\left\{\left|a_{m}-a_{k}\right|-R_{m}\right\}, k=1, \ldots, n, \\
& R_{0}>\rho>R_{0}^{0}=\max _{1 \leqslant m \leqslant n-1}\left\{R_{m}+\left|a_{m}\right|\right\} .
\end{aligned}
$$

Причем

$$
\left.\operatorname{Var}\left(\sigma_{m}^{(p)}(\varphi)\right)\right|_{[-\pi, \pi]}<L,\left.\quad \operatorname{Var}\left(\tau_{m}^{(p)}(\varphi)\right)\right|_{[-\pi, \pi]}<L, \quad m=0,1, \ldots, n .
$$

Полагая в (1.20) $р$ стремящимся $\mathrm{K}+\infty$, нетрудно убедиться на основании теоремы Хелли, что имеет место формула

$$
U(r, \vartheta)=\mathscr{A}_{0}+\sum_{k=0}^{n} \frac{\delta_{k}}{2 \pi} \int_{-\pi}^{\pi} \operatorname{Re} H_{k}\left(a_{k}+R_{k} \exp (i \varphi), r \exp (i \vartheta)\right) d \mu_{k}(\varphi),
$$

где

$$
\mu_{k}(\varphi) \in \mathrm{VB}[-\pi, \pi], \quad k=0,1, \ldots, n
$$

причем

$$
\begin{aligned}
\mathscr{A}_{m} & =\frac{1}{2 \pi} \int_{-\pi}^{\pi} d \mu_{m}(\varphi)=\sum_{\substack{k=0 \\
k \neq m}}^{n} \frac{\delta_{k}}{2 \pi} \int_{-\pi}^{\pi} S_{k}\left(a_{k}+R_{k} \exp (i \varphi)\right) d \mu_{k}(\varphi) \\
& =\frac{1}{2 \pi} \int_{-\pi}^{\pi} U_{m}(\rho, \varphi) d \varphi=\sum_{\substack{k=0 \\
k \neq m}}^{n} \frac{\delta_{k}}{2 \pi} \int_{-\pi}^{\pi} U_{k}(\rho, \varphi) S_{k}\left(a_{k}+R_{k} \exp (i \varphi)\right) d \varphi
\end{aligned}
$$

$m=1, \ldots, n$

$$
\begin{aligned}
& R_{k}<\rho<R_{k}^{0}=\min _{\substack{1 \leqslant m \leqslant n \\
m \neq k}}\left\{\left|a_{m}-a_{k}\right|-R_{m}\right\}, k=1, \ldots, n, \\
& R_{0}>\rho>R_{0}^{0}=\max _{1 \leqslant m \leqslant n-1}\left\{R_{m}+\left|a_{m}\right|\right\} .
\end{aligned}
$$

Здесь $r \exp (i \vartheta) \in \mathscr{K}\left(R_{j} r_{0}^{\delta_{j}}, a_{j}, j=0, \ldots, n\right)$.

Так как $r_{0}$ можно считать сколь угодно близким к единице, то формула (1.20) доказана для всей круговой конечносвязной области $\mathscr{K}\left(R_{j}, a_{j}, j=0, \ldots, n\right)$. Отсюда следует, что и формула (1.11) тоже установлена, так как известно, что при совпадении вещественных частей двух аналитических функций в некоторой области эти функции совпадают в этой области с точностью до чисто мнимой постоянной. 
СлЕДСТвИЕ 1.1.1. Если в условиях теоремъ 1.1 предположить, что функиии $\mu(\vartheta), \widetilde{\mu}_{k}(\vartheta), k=0, \ldots, n$, непрерьвно дифференцируемы на сегменте $[-\pi, \pi]$, то формула (1.11) превращается в формулу Шварца для конечносвязной круговой области [1], [2].

СлЕДСТвИЕ 1.1.2. Если в условиях теоремъ 1.1 положить $n=1$, то получаем известную теорему Зморовича [4], [5].

СлЕДСТВИЕ 1.1.3. Если в условиях теоремь 1.1 положить $n=0$, то получаем известную теорему Паатеро [6], [7].

\section{§2. Структурная формула для аналитических функций в классе $\mathscr{B}_{1}\left(R_{j}, a_{j}, j=0, \ldots, n\right)$}

Назовем функцией класса $\mathscr{B}_{N}\left(R_{j}, a_{j}, j=0, \ldots, n\right)$ каждую однозначную мероморфную в круговой конечносвязной области $\mathscr{K}\left(R_{j}, a_{j}, j=0, \ldots, n\right)$ функцию $f(z)$, имеюшую в этой круговой конечносвязной области конечное число полюсов не выше $N$-го порядка и принадлежашую классам $\mathscr{B}\left(R_{j}, \delta_{j}^{\prime}\right), j=1, \ldots, n$, $\mathscr{B}\left(\delta_{0}^{\prime}, R_{0}\right)$,

$$
\begin{aligned}
& R_{k}<\delta_{k}^{\prime}<R_{k}^{0}=\min _{\substack{1 \leqslant m \leqslant n \\
m \neq k}}\left\{\left|a_{m}-a_{k}\right|-R_{m}\right\}, k=1, \ldots, n, \\
& R_{0}>\delta_{0}^{\prime}>R_{0}^{0}=\max _{1 \leqslant m \leqslant n-1}\left\{R_{m}+\left|a_{m}\right|\right\},
\end{aligned}
$$

при достаточно малом $\delta_{j}^{\prime}-R_{j}>0, j=1, \ldots, n, R_{0}-\delta_{0}^{\prime}>0$.

Заметим, что

$$
\begin{aligned}
\mathscr{B}_{0}\left(R_{j}, a_{j}, j\right. & =0, \ldots, n) \\
\mathscr{B}_{M}\left(R_{j}, a_{j}, j=0, \ldots, n\right) & \supset \mathscr{B}_{N}\left(R_{j}, a_{j}, j=0, \ldots, n\right),
\end{aligned}
$$

TEOPEMA 2.1. Пусть

$$
\omega_{m}\left(z ; \alpha_{p}, \mathscr{C}_{p}\right)=\frac{1}{2}\left(\overline{\overline{\mathscr{C}_{p}}} \overline{\overline{\alpha_{p}}} \overline{H_{m}}\left(\overline{\alpha_{p}}, L_{m}(z)\right)-\frac{\mathscr{C}_{p}}{\alpha_{p}} H_{m}\left(\alpha_{p}, z\right)\right)
$$

ฉде

$$
\begin{gathered}
H_{k}(\zeta, z)=\left.\sum_{\nu=0}^{\infty} \sum_{k_{1} \neq k_{2}}^{k_{2 \nu} \neq m} \sum_{m=0}^{n} \frac{\zeta+\overline{L_{k}} E_{2 \nu} L_{m}(z)}{\zeta-\overline{L_{k}} E_{2 \nu} L_{m}(z)}\right|_{b_{m}} ^{z}, \\
b_{0}=0, \quad b_{m}=\infty, \quad m=1, \ldots, n, \\
E_{2 \nu}(z)=L_{k_{1}} \overline{L_{k_{2}}} \cdots L_{k_{2 \nu}-1} \overline{L_{k_{2 \nu}}}(z), \quad \nu=1,2, \ldots, \\
E_{0}(z) \equiv z, \quad L_{k}(\zeta)=\overline{a_{k}}+\frac{R_{k}^{2}}{\zeta-a_{k}},
\end{gathered}
$$

a $\alpha_{p}$ и $\mathscr{C}_{p}, p=1, \ldots, N,-$ комплексные числа такие, что

$$
\begin{aligned}
& R_{k}<\left|\alpha_{p}\right|<R_{k}^{0}=\min _{\substack{1 \leqslant m \leqslant n \\
m \neq k}}\left\{\left|a_{m}-a_{k}\right|-R_{m}\right\}, \quad k=1, \ldots, n, \\
& R_{0}>\left|\alpha_{p}\right|>R_{0}^{0}=\max _{1 \leqslant m \leqslant n-1}\left\{R_{m}+\left|a_{m}\right|\right\}, \quad p=1, \ldots, N .
\end{aligned}
$$


Причем

$$
\left.\operatorname{Re} \omega_{m}\left(z, \alpha_{p}, \mathscr{C}_{p}\right)\right|_{\left|z-a_{k}\right|=R_{k}}=\left\{\begin{array}{ll}
0, & k=m, \\
\lambda_{m, k}(p), & k \neq m,
\end{array} \quad \lambda_{m}=\sum_{p=1}^{N} \lambda_{0, m}(p) .\right.
$$

Далее, пусть $\widetilde{\mu}_{k}(\vartheta) \in \mathrm{VB}[-\pi, \pi], k=0,1, \ldots, n$, удовлетворяют соотношениям

$$
\begin{aligned}
\mathscr{A}_{m}=\frac{1}{2 \pi} \int_{-\pi}^{\pi} d \widetilde{\mu}_{m}(\vartheta)=\sum_{\substack{k=0 \\
k \neq m}}^{n} \frac{\delta_{k}}{2 \pi} \int_{-\pi}^{\pi} S_{k}\left(a_{k}\right. & \left.+R_{k} \exp (i \vartheta)\right) d \widetilde{\mu}_{k}(\vartheta)-\operatorname{Re} \lambda_{m} \\
& +\sum_{\substack{k=0 \\
k \neq m}}^{n} \operatorname{Re} \lambda_{k} \beta_{k}, \quad m=1, \ldots, n,
\end{aligned}
$$

ฉдe

$$
\begin{aligned}
& S_{m}(\zeta)=1+\frac{1}{2} \sum_{\nu=0}^{\infty} \sum_{k_{1} \neq k_{2}}^{k_{2 \nu} \neq k} \sum_{\substack{k=0 \\
k \neq m}}^{n}\left(\left.\frac{\zeta+\overline{L_{m}} E_{2 \nu} L_{k}(z)}{\zeta-\overline{L_{m}} E_{2 \nu} L_{k}(z)}\right|_{b_{k}} ^{a_{m}}-\left.\frac{\zeta+\overline{E_{2 \nu} L_{k}}(z)}{\zeta-\overline{E_{2 \nu} L_{k}}(z)}\right|_{\overline{b_{k}}} ^{\overline{a_{m}}}\right), \\
& \beta_{k}=\frac{\delta_{k}}{2 \pi} \int_{\left|z-a_{k}\right|=\rho_{k}} S_{k}(z) d z \\
& R_{k}<\rho_{k}<R_{k}(1+\delta)<\min _{\substack{1 \leqslant m \leqslant n \\
m \neq k}}\left\{\left|a_{m}-a_{k}\right|-R_{m}\right\}, \quad k=1, \ldots, n, \\
& R_{0}>\rho_{0}>R_{0}(1-\delta)>\max _{1 \leqslant m \leqslant n-1}\left\{R_{m}+\left|a_{m}\right|\right\} .
\end{aligned}
$$

Кроме того,

$$
\begin{gathered}
\mathscr{A}_{0}+i \mathscr{D}=\frac{1}{2 \pi i} \int_{|z|=\rho} \frac{f(z)}{z} d z, \\
R_{0}>\rho>R_{0}(1-\delta)>\max _{1 \leqslant m \leqslant n-1}\left\{R_{m}+\left|a_{m}\right|\right\},
\end{gathered}
$$

где $\delta>0$ и достаточно мало.

Тогда структурная формула

$$
\begin{aligned}
f(z)=\sum_{p=1}^{N} \omega_{0}\left(z ; \alpha_{p}, \mathscr{C}_{p}\right)+\sum_{k=0}^{n} \frac{\delta_{k}}{2 \pi} \int_{-\pi}^{\pi} H_{k}\left(a_{k}+\right. & \left.R_{k} \exp (i \vartheta), z\right) d \widetilde{\mu}_{k}(\vartheta) \\
& +\frac{1}{2 \pi} \int_{-\pi}^{\pi} d \widetilde{\mu}_{0}(\vartheta)+i \mathscr{D},
\end{aligned}
$$

где $N$ - число полюсов функции $f(z)$ в круговой конечносвязной области $\mathscr{K}\left(R_{j}, a_{j}, j=0, \ldots, n\right)$, является необходимым и достаточныц условием принадлежности функции $f(z)$ классу $\mathscr{B}_{1}\left(R_{j}, a_{j}, j=0, \ldots, n\right)$. 
ДокаЗАтеЛЬСтво. Достаточность. Так как функция $\omega_{0}\left(z ; \alpha_{p}, \mathscr{C}_{p}\right), \alpha_{p} \in$ $\mathscr{K}\left(R_{j}, a_{j}, j=0, \ldots, n\right), p=1, \ldots, n$, мероморфна в круговой конечносвязной области $\mathscr{K}\left(R_{j}, a_{j}, j=0, \ldots, n\right)$ и имеет в этой области только один полюс, а именно, простой полюс в точке $\alpha_{p}$ с вычетом $\mathscr{C}_{p}[9, \S 5$, п. 23] и так как функция

$$
\Psi(z)=f(z)-\sum_{p=1}^{N} \omega_{0}\left(z ; \alpha_{p}, \mathscr{C}_{p}\right)
$$

в силу теоремы 1.1 принадлежит классу $\mathscr{B}\left(R_{j}, a_{j}, j=0, \ldots, n\right)$, то $f(z) \in$ $\mathscr{B}_{1}\left(R_{j}, a_{j}, j=0, \ldots, n\right)$.

Необходимость. Пусть полюсами функции $f(z)$ класса $\mathscr{B}_{1}\left(R_{j}, a_{j}, j=0, \ldots, n\right)$ и ее вычетами относительно этих полюсов будут, соответственно, $\alpha_{p}$ и $\mathscr{C}_{p}$, где

$$
\begin{aligned}
& R_{k}<\left|\alpha_{p}\right|<R_{k}^{0}=\min _{\substack{1 \leqslant m \leqslant n \\
m \neq k}}\left\{\left|a_{m}-a_{k}\right|-R_{m}\right\}, \quad k=1, \ldots, n, \\
& R_{0}>\left|\alpha_{p}\right|>R_{0}^{0}=\max _{1 \leqslant m \leqslant n-1}\left\{R_{m}+\left|a_{m}\right|\right\}, \quad p=1, \ldots, N .
\end{aligned}
$$

Образуем функцию особенностей

$$
\Phi(z)=\sum_{p=1}^{N} \omega_{0}\left(z ; \alpha_{p}, \mathscr{C}_{p}\right)
$$

Введем обозначение

$$
\Psi(z)=f(z)-\Phi(z) .
$$

Легко видеть, что $\Psi(z) \in \mathscr{B}_{0}\left(R_{j}, a_{j}, j=0, \ldots, n\right)$. Тогда по теореме 1.1 будем иметь

$$
f(z)=\Phi(z)+\sum_{k=0}^{n} \frac{\delta_{k}}{2 \pi} \int_{-\pi}^{\pi} H_{k}\left(a_{k}+R_{k} \exp (i \vartheta), z\right) d \mu_{k}^{*}(\vartheta)+\mathscr{A}_{0}^{*}+i \mathscr{D}^{*}
$$

где

$$
\mu_{k}^{*}(\vartheta) \in \mathrm{VB}[-\pi, \pi], \quad k=0,1, \ldots, n
$$

Причем

$$
\begin{aligned}
\mathscr{A}_{m}^{*} & =\frac{1}{2 \pi} \int_{-\pi}^{\pi} d \mu_{m}^{*}(\vartheta)=\sum_{\substack{k=0 \\
k \neq m}}^{n} \frac{\delta_{k}}{2 \pi} \int_{-\pi}^{\pi} S_{k}\left(a_{k}+R_{k} \exp (i \vartheta)\right) d \mu_{k}^{*}(\vartheta) \\
& =\frac{1}{2 \pi} \int_{-\pi}^{\pi} \operatorname{Re} \Psi\left(a_{m}+\rho \exp (i \varphi)\right) d \varphi \\
& =\sum_{\substack{k=0 \\
k \neq m}}^{n} \frac{\delta_{k}}{2 \pi} \int_{-\pi}^{\pi} \operatorname{Re} \Psi\left(a_{k}+\rho \exp (i \varphi)\right) S_{k}\left(a_{k}+R_{k} \exp (i \varphi)\right) d \varphi, \quad m=1, \ldots, n,
\end{aligned}
$$




$$
\begin{aligned}
& R_{k}<\rho<R_{k}^{0}=\min _{1 \leqslant m}\left\{\left|a_{m}-a_{k}\right|-R_{m}\right\}, k=1, \ldots, n, \\
& R_{0}>\rho>R_{0}^{0}=\max _{1 \leqslant m \leqslant n-1}\left\{R_{m}+\left|a_{m}\right|\right\},
\end{aligned}
$$

где

$$
S_{m}(\zeta)=1+\frac{1}{2} \sum_{\nu=0}^{\infty} \sum_{k_{1} \neq k_{2}}^{k_{2 \nu} \neq k} \sum_{\substack{k=0 \\ k \neq m}}^{n}\left(\left.\frac{\zeta+\overline{L_{m}} E_{2 \nu} L_{k}(z)}{\zeta-\overline{L_{m}} E_{2 \nu} L_{k}(z)}\right|_{b_{k}} ^{a_{m}}-\left.\frac{\zeta+\overline{E_{2 \nu} L_{k}}(z)}{\zeta-\overline{E_{2 \nu} L_{k}}(z)}\right|_{\overline{b_{k}}} ^{\overline{a_{m}}}\right) .
$$

Кроме того,

$$
\mathscr{A}_{0}^{*}+i \mathscr{D}^{*}=\frac{1}{2 \pi i} \int_{|z|=\rho} \frac{\Psi(z)}{z} d z, \quad R_{0}>\rho>R_{0}^{0}=\max _{1 \leqslant m \leqslant n-1}\left\{R_{m}+\left|a_{m}\right|\right\} .
$$

Формула (2.2) и есть искомая.

Заметим, что

$$
\begin{aligned}
\operatorname{Re} \Phi\left(R_{0} \exp (i \varphi)\right) & =0, \\
\operatorname{Re} \Phi\left(a_{k}+R_{k} \exp (i \varphi)\right)=-\operatorname{Re} \lambda_{k}, \quad k & =1, \ldots, n, \quad-\pi \leqslant \varphi \leqslant \pi .
\end{aligned}
$$

Тогда

$$
\begin{aligned}
\mathscr{A}_{m}^{*}+i \mathscr{D}_{m}^{*} & =\frac{1}{2 \pi i} \int_{\left|z-a_{m}\right|=\rho_{m}} \frac{f(z)}{z-a_{m}} d z+\operatorname{Re} \lambda_{m}+i \operatorname{Im} \lambda_{m} \\
& =\sum_{\substack{k=0 \\
k \neq m}}^{n} \frac{\delta_{k}}{2 \pi} \int_{\left|z-a_{k}\right|=\rho_{k}} \frac{f(z)}{z-a_{k}} S_{k}(z) d z+\sum_{\substack{k=0 \\
k \neq m}}^{n}\left(\operatorname{Re} \lambda_{k}+i \operatorname{Im} \lambda_{k}\right) \beta_{k},
\end{aligned}
$$

где

$$
\begin{gathered}
\beta_{k}=\frac{\delta_{k}}{2 \pi} \int_{\left|z-a_{k}\right|=\rho_{k}} S_{k}(z) d z, \\
R_{k}<\rho_{k}<R_{k}(1+\delta)<\min _{\substack{1 \leqslant m \leqslant n \\
m \neq k}}\left\{\left|a_{m}-a_{k}\right|-R_{m}\right\}, \quad k=1, \ldots, n, \\
R_{0}>\rho_{0}>R_{0}(1-\delta)>\max _{1 \leqslant m \leqslant n-1}\left\{R_{m}+\left|a_{m}\right|\right\},
\end{gathered}
$$

причем $\delta>0$ и достаточно мало.

Положим

$$
\begin{gathered}
\mu_{m}^{*}(\vartheta)=\mu_{m}(\vartheta)+(\pi+\varphi)\left(\operatorname{Re} \lambda_{m}-\sum_{\substack{k=0 \\
k \neq m}}^{n} \operatorname{Re} \lambda_{k} \beta_{k}\right), m=1, \ldots, n, \\
\mu_{0}^{*}(\vartheta)=\mu_{0}(\vartheta), \\
\mathscr{A}_{m}=\mathscr{A}_{m}^{*}-\operatorname{Re} \lambda_{m}+\sum_{\substack{k=0 \\
k \neq m}}^{n} \operatorname{Re} \lambda_{k} \beta_{k}, \\
\mathscr{D}_{m}=\mathscr{D}_{m}^{*}-\operatorname{Im} \lambda_{m}+\sum_{\substack{k=0 \\
k \neq m}}^{n} \operatorname{Im} \lambda_{k} \beta_{k} .
\end{gathered}
$$


Тогда формула (2.2) принимает вид (2.1).

Возникает вопрос о единственности интегрального представления (2.1). Так как по заданной вешественной части функции $f(z)$ регулярная в круговой конечносвязной области $\mathscr{K}\left(R_{j}, a_{j}, j=0, \ldots, n\right)$ функция $f(z)-\Phi(z)-\mathscr{A}_{0}-i \mathscr{D}$ определяется единственным образом, то функции $\mu_{m}(\varphi), m=0,1, \ldots, n$, определены с точностью до их значений в точках разрыва, причем эта неопределенность устраняется соответствуюшей нормировкой этих функций в их точках разрьва.

СледСТвиЕ 2.1.1. Если в условиях теоремы 2.1 предположить, что функиии $\widetilde{\mu}_{k}(\vartheta), k=0, \ldots, n$, непрерывно дифференцируемы на сегменте $[-\pi, \pi]$, то формула (2.1) превращается в обобщение формуль ШІварца для конечносвязной круговой области на мероморфные функции, обладающие только простыми полюсами.

СлЕДСТвИЕ 2.1.2. Если в условиях теоремы 2.1 положить $n=1$, то получаем известную теорему Зморовича [5].

\section{§3. Структурная формула для мероморфных аналитических функций в $\mathscr{K}\left(R_{j}, a_{j}, j=0, \ldots, n\right)$}

Назовем функцией класса $\mathscr{B}^{*}\left(R_{j}, a_{j}, j=0, \ldots, n\right)$ каждую однозначную мероморфную в круговой конечносвязной области $\mathscr{K}\left(R_{j}, a_{j}, j=0, \ldots, n\right)$ функцию $f(z)$, удовлетворяюшую условию

$$
f_{1}(z) \equiv \frac{z f^{\prime}(z)}{f(z)} \in \mathscr{B}_{1}\left(R_{j}, a_{j}, j=0, \ldots, n\right) .
$$

Назовем функиией класса $\mathscr{B}^{0}\left(R_{j}, a_{j}, j=0, \ldots, n\right)$ каждую однозначную мероморфную в круговой конечносвязной области $\mathscr{K}\left(R_{j}, a_{j}, j=0, \ldots, n\right)$ функцию $f(z)$, удовлетворяюшую условию

$$
f_{2}(z) \equiv 1+\frac{z f^{\prime \prime}(z)}{f^{\prime}(z)} \in \mathscr{B}_{1}\left(R_{j}, a_{j}, j=0, \ldots, n\right) .
$$

Имеют место теоремы.

ТеОрема 3.1. Пусть $\mu_{k}(\vartheta), k=0,1, \ldots, n,-$ функиии класса $\mathrm{VB}[-\pi, \pi]$, удовлетворяющие соотношениям

$$
\begin{gathered}
\mathscr{A}_{m}=\frac{1}{2 \pi} \int_{-\pi}^{\pi} d \mu_{m}(\vartheta)=\sum_{\substack{k=0 \\
k \neq m}}^{n} \frac{\delta_{k}}{2 \pi} \int_{-\pi}^{\pi} S_{k}\left(a_{k}+R_{k} \exp (\imath \vartheta)\right) d \mu_{k}(\vartheta) \\
-\lambda_{m}+\sum_{\substack{k=0 \\
k \neq m}}^{n} \lambda_{k} \beta_{k}^{\prime}, \quad m=1, \ldots, n,
\end{gathered}
$$

ฉдe

$$
\lambda_{m}=-\left.\operatorname{Re}\left(\sum_{j=1}^{N_{0}} \omega_{0}\left(\zeta ; \alpha_{j}, p_{j} \alpha_{j}\right)+\sum_{k=1}^{N_{p}} \omega_{0}\left(\zeta ; \beta_{k},-s_{k} \beta_{k}\right)\right)\right|_{\left|\zeta-a_{m}\right|=R_{m}}
$$


Здеcъ

$$
\omega_{m}\left(z ; \alpha_{p}, \mathscr{C}_{p}\right)=\frac{1}{2}\left(\overline{\frac{\mathscr{C}_{p}}{\overline{\alpha_{p}}}} \overline{H_{m}}\left(\overline{\alpha_{p}}, L_{m}(z)\right)-\frac{\mathscr{C}_{p}}{\alpha_{p}} H_{m}\left(\alpha_{p}, z\right)\right)
$$

əдe

$$
\begin{gathered}
H_{k}(\zeta, z)=\left.\sum_{\nu=0}^{\infty} \sum_{k_{1} \neq k_{2}}^{k_{2 \nu} \neq m} \sum_{m=0}^{n} \frac{\zeta+\overline{L_{k}} E_{2 \nu} L_{m}(z)}{\zeta-\overline{L_{k}} E_{2 \nu} L_{m}(z)}\right|_{b_{m}} ^{z}, \\
b_{0}=0, \quad b_{m}=\infty, \quad m=1, \ldots, n, \\
\beta_{k}^{\prime}=\frac{\delta_{k}}{2 \pi} \int_{\left|z-a_{k}\right|=\rho_{k}} S_{k}(z) d z,
\end{gathered}
$$

$\delta_{k}-$ символ Кронекера, $p_{j}, j=1, \ldots, N_{0}, u s_{k}, k=1, \ldots, N_{p},-$ челье, неотрицательные числа.

Причем

$$
S_{m}(\zeta)=1+\frac{1}{2} \sum_{\nu=0}^{\infty} \sum_{k_{1} \neq k_{2}}^{k_{2 \nu} \neq k} \sum_{\substack{k=0 \\ k \neq m}}^{n}\left(\left.\frac{\zeta+\overline{L_{m}} E_{2 \nu} L_{k}(z)}{\zeta-\overline{L_{m}} E_{2 \nu} L_{k}(z)}\right|_{b_{k}} ^{a_{m}}-\left.\frac{\zeta+\overline{E_{2 \nu} L_{k}}(z)}{\zeta-\overline{E_{2 \nu} L_{k}}(z)}\right|_{\overline{b_{k}}} ^{\overline{a_{m}}}\right) .
$$

При этом

$$
\begin{gathered}
L_{k}(\zeta)=\overline{a_{k}}+\frac{R_{k}^{2}}{\zeta-a_{k}}, \\
E_{2 \nu}(z)=L_{k_{1}} \overline{L_{k_{2}}} \cdots L_{k_{2 \nu-1}} \overline{L_{k_{2 \nu}}}(z), \quad \nu=1,2, \ldots, \\
E_{0}(z) \equiv z
\end{gathered}
$$

Тогда структурная формула

$$
f(z)=C z^{\alpha+\beta+i \mathscr{D}} Q(z) \exp \left(\frac{1}{2 \pi} S(z)\right)
$$

где $C$ - комплексная постоянная, $\alpha$ - целое число, является необходимыцм и достаточныц условием принадлежнности функции $f(z)$ классу $\mathscr{B}^{*}\left(R_{j}, a_{j}\right.$, $j=0, \ldots, n)$.

Здесь

$$
\beta=\sum_{\kappa=0}^{n} \delta_{\kappa} \int_{-\pi}^{\pi} \sum_{\nu=0}^{\infty} \sum_{k_{1} \neq k_{2}}^{k_{2 \nu} \neq k} \sum_{k=0}^{n} A_{\kappa, \nu, k}\left(a_{k}+R_{k} \exp (i \vartheta)\right) d \mu_{\kappa}(\vartheta),
$$

əде

$$
A_{\kappa, \nu, k}(\zeta)=\frac{2\left(\zeta-a_{\kappa}\right)\left(N_{\kappa, \nu, k}(0)-N_{\kappa, \nu, k}\left(b_{k}\right)\right)}{\left(\zeta-N_{\kappa, \nu, k}(0)\right)\left(\zeta-N_{\kappa, \nu, k}\left(b_{k}\right)\right)}
$$

причем

$$
N_{k, \nu, m}(z)=\overline{L_{k}} E_{2 \nu} L_{m}(z)
$$


При этом

$$
Q(z)=\prod_{\nu=0}^{\infty} \prod_{k_{1} \neq k_{2}}^{k_{2 \nu} \neq k} \prod_{k=0}^{n}\left(\frac{G_{m, \nu, k}\left(z, N_{0}\right)}{G_{m, \nu, k}\left(z, N_{p}\right)}\right)
$$

$2 \partial e$

$$
\begin{aligned}
G_{m, \nu, k}(z, N) & =E_{m, \nu, k}(z, N) z^{F_{m, \nu, k}(N)} \\
E_{m, \nu, k}\left(z ; \zeta, p_{j}\right) & =\frac{\left(z-\overline{L_{m} M_{k, \nu, m}}(\bar{\zeta})\right)^{\overline{p_{j}} D_{m, \nu, k}(\zeta)}}{\left(z-M_{k, \nu, m}(\zeta)\right)^{p_{j} B_{m, \nu, k}(\zeta)}}
\end{aligned}
$$

Кроме того,

$$
\begin{aligned}
S(z)=\sum_{\kappa=0}^{n} \delta_{\kappa} \int_{-\pi}^{\pi} \sum_{\nu=0}^{\infty} \sum_{k_{1} \neq k_{2}}^{k_{2 \nu} \neq k} \sum_{k=0}^{n} B_{\kappa, \nu, k}\left(a_{k}+R_{k} \exp (i \vartheta)\right) \\
\times \ln \left(z-M_{k, \nu, \kappa}\left(a_{k}+R_{k} \exp (i \vartheta)\right)\right) d \mu_{\kappa}(\vartheta)
\end{aligned}
$$

$2 \partial e$

$$
\begin{aligned}
B_{\kappa, \nu, k}(\zeta) & =\frac{2\left(\zeta-a_{\kappa}\right)\left(N_{\kappa, \nu, k}(\infty)-N_{\kappa, \nu, k}(0)\right)}{\left(\zeta-N_{\kappa, \nu, k}(0)\right)\left(\zeta-N_{\kappa, \nu, k}(\infty)\right)} \\
M_{m, \nu, k}(z) & =\overline{L_{m}} L_{k_{2 \nu}} \overline{L_{k_{2 \nu}-1}} \cdots L_{k_{2}} \overline{L_{k_{1}}} L_{k}(z) .
\end{aligned}
$$

ДоказАтельство. Достаточность. Вычисляя функцию $f_{1}(z) \equiv \frac{z f^{\prime}(z)}{f(z)}$ на основании (3.3) и учитывая, что

$$
H_{k}(\zeta, z)=\left.\sum_{\nu=0}^{\infty} \sum_{k_{1} \neq k_{2}}^{k_{2 \nu} \neq m} \sum_{m=0}^{n} \frac{\zeta+\overline{L_{k}} E_{2 \nu} L_{m}(z)}{\zeta-\overline{L_{k}} E_{2 \nu} L_{m}(z)}\right|_{b_{m}} ^{z}
$$

получаем формулу $(2.1)$, когда $\mathscr{D}=0$. Отсюда следует, что $f_{1}(z) \in \mathscr{B}_{1}\left(R_{j}, a_{j}\right.$, $j=0, \ldots, n)$ и $f(z) \in \mathscr{B}^{*}\left(R_{j}, a_{j}, j=0, \ldots, n\right)$.

Необходимость. Обозначая через $\alpha_{j}, j=1, \ldots, N_{0}$, нули, а через $\beta_{k}$, $k=1, \ldots, N_{p}$, - полюсы функции $f(z)$ в круговой конечносвязной области $\mathscr{K}\left(R_{j}, a_{j}, j=0, \ldots, n\right)$, через $p_{j}$ и $s_{k}-$ кратности $\alpha_{j}$ и $\beta_{k}$, соответственно, получаем для $\frac{z f^{\prime}(z)}{f(z)}$ интегральное представление

$$
\begin{aligned}
& \frac{z f^{\prime}(z)}{f(z)}=\sum_{j=1}^{N_{0}} \omega_{0}\left(z ; \alpha_{j}, p_{j} \alpha_{j}\right)+\sum_{k=1}^{N_{p}} \omega_{0}\left(z ; \beta_{k},-s_{k} \beta_{k}\right) \\
& \quad+\sum_{k=0}^{n} \frac{\delta_{k}}{2 \pi} \int_{-\pi}^{\pi} H_{k}\left(a_{k}+R_{k} \exp (i \vartheta), z\right) d \mu_{k}(\vartheta)+\frac{1}{2 \pi} \int_{-\pi}^{\pi} d \mu_{0}(\vartheta)+i \mathscr{D},
\end{aligned}
$$

где

$$
\mu_{k}(\vartheta) \in \mathrm{VB}[-\pi, \pi], \quad k=0,1, \ldots, n .
$$


Причем

$$
\begin{gathered}
\mathscr{A}_{m}=\frac{1}{2 \pi} \int_{-\pi}^{\pi} d \mu_{m}(\vartheta)=\sum_{\substack{k=0 \\
k \neq m}}^{n} \frac{\delta_{k}}{2 \pi} \int_{-\pi}^{\pi} S_{k}\left(a_{k}+R_{k} \exp (i \vartheta)\right) d \mu_{k}(\vartheta) \\
-\lambda_{m}+\sum_{\substack{k=0 \\
k \neq m}}^{n} \lambda_{k} \beta_{k}^{\prime}, \quad m=1, \ldots, n,
\end{gathered}
$$

где

$$
\begin{aligned}
\beta_{k}^{\prime} & =\frac{\delta_{k}}{2 \pi} \int_{\left|z-a_{k}\right|=\rho_{k}} S_{k}(z) d z \\
\lambda_{m} & =-\left.\operatorname{Re}\left(\sum_{j=1}^{N_{0}} \omega_{0}\left(\zeta ; \alpha_{j}, p_{j} \alpha_{j}\right)+\sum_{k=1}^{N_{p}} \omega_{0}\left(\zeta ; \beta_{k},-s_{k} \beta_{k}\right)\right)\right|_{\left|\zeta-a_{m}\right|=R_{m}} \\
& =\sum_{j=1}^{N_{0}} p_{j}-\sum_{k=1}^{N_{p}} s_{k} .
\end{aligned}
$$

Кроме того,

$$
\begin{gathered}
\mathscr{A}_{0}+i \mathscr{D}=\frac{1}{2 \pi i} \int_{|z|=\rho} \frac{f(z)}{z} d z, \\
R_{0}>\rho>R_{0}(1-\delta)>\max _{1 \leqslant m \leqslant n-1}\left\{R_{m}+\left|a_{m}\right|\right\},
\end{gathered}
$$

где $\delta>0$ и достаточно мало.

Пусть при любом натуральном $\nu$

$$
\begin{aligned}
A_{\kappa, \nu, k}(\zeta) & =\frac{2\left(\zeta-a_{\kappa}\right)\left(N_{\kappa, \nu, k}(0)-N_{\kappa, \nu, k}\left(b_{k}\right)\right)}{\left(\zeta-N_{\kappa, \nu, k}(0)\right)\left(\zeta-N_{\kappa, \nu, k}\left(b_{k}\right)\right)}, \\
B_{\kappa, \nu, k}(\zeta) & =\frac{2\left(\zeta-a_{\kappa}\right)\left(N_{\kappa, \nu, k}(\infty)-N_{\kappa, \nu, k}(0)\right)}{\left(\zeta-N_{\kappa, \nu, k}(0)\right)\left(\zeta-N_{\kappa, \nu, k}(\infty)\right)}, \\
C_{\kappa, \nu, k}(\zeta) & =\frac{2\left(\bar{\zeta}-\overline{a_{\kappa}}\right)\left(\overline{N_{\kappa, \nu, k}} L_{\kappa}(0)-\overline{N_{\kappa, \nu, k}}\left(\overline{b_{k}}\right)\right)}{\left(\bar{\zeta}-\overline{N_{\kappa, \nu, k}} L_{\kappa}(0)\right)\left(\bar{\zeta}-\overline{N_{\kappa, \nu, k}}\left(\overline{b_{k}}\right)\right)}, \\
D_{\kappa, \nu, k}(\zeta) & =\frac{2\left(\bar{\zeta}-\overline{a_{\kappa}}\right)\left(\overline{N_{\kappa, \nu, k}} L_{\kappa}(\infty)-\overline{N_{\kappa, \nu, k}} L_{\kappa}(0)\right)}{\left(\bar{\zeta}-\overline{N_{\kappa, \nu, k}}(0)\right)\left(\bar{\zeta}-\overline{N_{\kappa, \nu, k}}(\infty)\right)} .
\end{aligned}
$$

Нам потребуется функция

$$
N_{k, \nu, m}(z)=\overline{L_{k}} E_{2 \nu} L_{m}(z)
$$

При этом

$$
E_{2 \nu}(z)=L_{k_{1}} \overline{L_{k_{2}}} \cdots L_{k_{2 \nu-1}} \overline{L_{k_{2 \nu}}}(z), \quad \nu=1,2, \ldots
$$

где

$$
L_{k}(z)=\overline{a_{k}}+\frac{R_{k}^{2}}{z-a_{k}} .
$$


Функция $M_{m, \nu, k}(z)$, обратная $N_{k, \nu, m}(z)$, имеет вид:

$$
M_{m, \nu, k}(z)=\overline{L_{m}} L_{k_{2 \nu}} \overline{L_{k_{2 \nu-1}}} \cdots L_{k_{2}} \overline{L_{k_{1}}} L_{k}(z) .
$$

Из формул (3.12)-(3.15) следует

$$
\begin{aligned}
& N_{k, \nu, m}(z)=N_{k, \nu, m}(\infty) \frac{z-M_{m, \nu, k}(0)}{z-M_{m, \nu, k}(\infty)}, \\
& M_{m, \nu, k}(z)=M_{m, \nu, k}(\infty) \frac{z-N_{k, \nu, m}(0)}{z-N_{k, \nu, m}(\infty)}
\end{aligned}
$$

$\mathrm{C}$ целью более компактной записи сумм функций, определенных в $\mathscr{D}_{m}$, будем использовать следующие обозначения:

$$
\begin{aligned}
E_{m, \nu, k}\left(z ; \zeta, p_{j}\right) & =\frac{\left(z-\overline{L_{m} M_{k, \nu, m}}(\bar{\zeta})\right)^{\overline{p_{j}} D_{m, \nu, k}(\zeta)}}{\left(z-M_{k, \nu, m}(\zeta)\right)^{p_{j} B_{m, \nu}(\zeta)}} \\
F_{m, \nu, k}\left(\zeta ; p_{j}\right) & =\overline{p_{j}} C_{m, \nu, k}(\zeta)-p_{j} A_{m, \nu, k}(\zeta) \\
F_{m, \nu, k}\left(N_{0}\right) & =\sum_{j=1}^{N_{0}} F_{m, \nu, k}\left(\alpha_{j}, p_{j}\right) \\
F_{m, \nu, k}\left(N_{p}\right) & =\sum_{h=1}^{N_{p}} F_{m, \nu, k}\left(\beta_{h}, s_{h}\right) \\
E_{m, \nu, k}\left(z ; N_{0}\right) & =\prod_{j=1}^{N_{0}} E_{m, \nu, k}\left(z ; \alpha_{j}, p_{j}\right) \\
E_{m, \nu, k}\left(z ; N_{p}\right) & =\prod_{h=1}^{N_{p}} E_{m, \nu, k}\left(z ; \beta_{h}, s_{h}\right)
\end{aligned}
$$

Умножая обе части равенства $(3.7)$ на $\frac{d z}{z}$, интегрируя и затем потенцируя, получаем

$$
f(z)=C z^{\mathscr{A}_{0}+\beta+i \mathscr{D}} Q(z) \exp \left(\frac{1}{2 \pi} S(z)\right),
$$

где $C$ - комплексная постоянная, $\mathscr{A}_{0}$ - целое число.

Функции $Q(z)$ и $S(z)$ определяются, соответственно, формулами

$$
Q(z)=\prod_{\nu=0}^{\infty} \prod_{k_{1} \neq k_{2}}^{k_{2 \nu} \neq k} \prod_{k=0}^{n}\left(\frac{G_{m, \nu, k}\left(z, N_{0}\right)}{G_{m, \nu, k}\left(z, N_{p}\right)}\right),
$$

где

$$
G_{m, \nu, k}(z, N)=E_{m, \nu, k}(z, N) z^{F_{m, \nu, k}(N)}
$$

и

$$
\begin{aligned}
S(z)=\sum_{\kappa=0}^{n} \delta_{\kappa} \int_{-\pi}^{\pi} \sum_{\nu=0}^{\infty} \sum_{k_{1} \neq k_{2}}^{k_{2 \nu} \neq k} \sum_{k=0}^{n} B_{\kappa, \nu, k}\left(a_{k}+R_{k} \exp (i \vartheta)\right) \\
\times \ln \left(z-M_{k, \nu, \kappa}\left(a_{k}+R_{k} \exp (i \vartheta)\right)\right) d \mu_{\kappa}(\vartheta)
\end{aligned}
$$




$$
\beta=\sum_{\kappa=0}^{n} \delta_{\kappa} \int_{-\pi}^{\pi} \sum_{\nu=0}^{\infty} \sum_{k_{1} \neq k_{2}}^{k_{2 \nu} \neq k} \sum_{k=0}^{n} A_{\kappa, \nu, k}\left(a_{k}+R_{k} \exp (i \vartheta)\right) d \mu_{\kappa}(\vartheta),
$$

где $A_{\kappa, \nu, k}(\zeta)$ и $B_{\kappa, \nu, k}(\zeta)$ определяются, соответственно, формулами $(3.8),(3.9)$.

Так как функция $f(z)$ однозначна в круговой конечносвязной области $\mathscr{K}\left(R_{j}, a_{j}, j=0, \ldots, n\right)$, то $\mathscr{D}=0$. Теорема доказана.

СлЕДСТВИЕ 3.1.1. Если в условиях теоремы 3.1 положить $R_{0}=1, n=1$, то получаем известную теорему Зморовича [4].

Теорема 3.2. Структурная формула

$$
f(z)=C \int z^{\alpha-1} Q(z) \exp \left(\frac{1}{2 \pi} S(z)\right) d z+C_{1},
$$

где $C$ и $C_{1}$ - комплексные постоянные, $\alpha$ - челое число, является необходимым и достаточным условием принадлежности функции $f(z)$ классу $\mathscr{B}^{0}\left(R_{j}, a_{j}, j=0, \ldots, n\right)$.

Здесъ функции $Q(z), S(z)$ определяются, соответственно, формулами (3.22), (3.23).

Доказательство теоремы 3.2 проводится аналогично доказательству теоремы 3.1 с учетом (3.2).

СлЕДСТвИЕ 3.2.1. Если в условиях теоремы 3.2 положить $R_{0}=1, n=1$, то получаем известную теорему Зморовича [4].

Между постоянньми, входящими в формулу (3.7), сушествуют дополнительные соотношения (см. $\S 4,(4.27))$, обеспечиваюшие равенство нулю вычетов подинтегральной функции относительно $b_{p}, p=0,1, \ldots, N$.

Ограничимся рассмотрением только регулярных в круговой конечносвязной области $\mathscr{K}\left(R_{j}, a_{j}, j=0, \ldots, n\right)$ функций $f(z)$, для которых $\alpha \geqslant 1$, и обозначим множества таких функций, входящие в классы $\mathscr{B}^{*}\left(R_{j}, a_{j}, j=0, \ldots, n\right)$ и $\mathscr{B}^{0}\left(R_{j}, a_{j}, j=0, \ldots, n\right)$, соответственно, через $\widetilde{\mathscr{B}}^{*}\left(R_{j}, a_{j}, j=0, \ldots, n\right)$ и $\widetilde{\mathscr{B}}^{0}\left(R_{j}, a_{j}, j=0, \ldots, n\right)$.

Из теорем 3.1 и 3.2 следуют теоремы.

Теорема 3.3. Если однозначная мероморфная в круговой конечносвязной области $\mathscr{K}\left(R_{j}, a_{j}, j=0, \ldots, n\right)$ функция $f(z)$ принадлежит класcу $\widetilde{\mathscr{B}}^{*}\left(R_{j}, a_{j}, j=0, \ldots, n\right)$, то функция $\int \frac{f(z)}{z} d z$ принадлежсит классу $\widetilde{\mathscr{B}}^{0}\left(R_{j}, a_{j}, j=0, \ldots, n\right)$.

СлЕДСТВИЕ 3.3.1. Если в условиях теоремы 3.3 положить $R_{0}=1, n=1$, то получаем известную теорему Зморовича [4].

СЛЕДСТВИЕ 3.3.2. Если в условиях предыдущего следствия 3.3 .1 устремить $R_{1} к$ нулю, то получаем известную теорему Александера [6]. 
Теорема 3.4. Если регулярная функиия $f(z)$ принадлежит классу $\widetilde{\mathscr{B}}^{*}\left(R_{j}, a_{j}, j=0, \ldots, n\right), \quad$ то функция $\int \frac{f(z)}{z} d z$ принадлежит классу $\widetilde{\mathscr{B}}^{0}\left(R_{j}, a_{j}, j=0, \ldots, n\right)$.

СлЕДСТВИЕ 3.4.1. Если в условиях теоремы 3.4 положить $R_{0}=1, n=1$, то получаем известную теорему Зморовича [5].

СЛЕДСТВИЕ 3.4.2. Если в условиях предъдущего следствия 3.4 .1 устремить $R_{1}$ к нулю, то получаем известную теорему Александера [7].

Теорема 3.5. Если регулярная функиия $f(z)$ принадлежит класcy $\widetilde{\mathscr{B}}^{0}\left(R_{j}, a_{j}, j=0, \ldots, n\right), \quad$ то функиия $z f^{\prime}(z)$ принадлежит классу $\widetilde{\mathscr{B}}^{*}\left(R_{j}, a_{j}, j=0, \ldots, n\right)$.

СлЕДСТВИЕ 3.5.1. Если в условиях теоремы 3.5 положить $R_{0}=1, n=1$, то получаем известную теорему Зморовича [5].

СлЕДСТВИЕ 3.5.2. Если в условиях предыдущего следствия 3.5.1 устремить $R_{1} \kappa$ нулю, то получаем известную теорему Александера [7].

\section{§4. Построение решения задачи Шварца для мероморфных функций}

Построим решение задачи Шварца в классе функций, мероморфных в $(n+1)$-связной круговой области $\mathscr{K}$. Пусть заданы точки $b_{1}, b_{2}, \ldots, b_{n}$, где функция $f^{* *}(z)$ имеет кратные полюсы, кратностей, соответственно, $N_{1}, N_{2}, \ldots, N_{n}$.

Нахождение такой функции $f^{* *}(z)$ по известным значениям ее вешественной части $f_{k}^{* *}(\zeta)$ на граничных компонентах $\mathscr{C}_{k}(\zeta):\left|\zeta-a_{k}\right|=R_{k}, k=0,1, \ldots, n$, сведем к решению специальных систем уравнений, используя возможность представить функцию $f^{* *}(z)$ в следующем виде $[9$, гл. $3, \S 1$, п. 42$]$ :

$$
f^{* *}(z)=M+\sum_{k=0}^{n} \varphi_{k}(z)+\sum_{j=1}^{H} \sum_{p=1}^{N_{j}} \frac{B_{p}^{j}}{\left(z-b_{j}\right)^{p}},
$$

где $M, B_{p}^{j}, j=1, \ldots, H, p=1, \ldots, N_{j}$, - некоторые постоянные, $\varphi_{k}(z)$ - регулярная функция в $\mathscr{D}_{k}, k=0,1, \ldots, n$.

В силу принятых обозначений, на граничных компонентах $\mathscr{C}_{k}(\zeta)$ области $\mathscr{K}$ имеют место соотношения

$$
\operatorname{Re} f^{* *}(\zeta)=f_{m}^{* *}(\zeta), \quad \zeta \in \mathscr{C}_{m}(\zeta), \quad m=0,1, \ldots, n
$$

Обратимся к выводу специальных систем уравнений для определения функций $\varphi_{k}(z), k=0,1, \ldots, n$.

Умножим обе части равенства (4.2) на

$$
\frac{1}{2 \pi i} \frac{d \zeta}{\zeta-z}
$$

и проинтегрируем произведение по $\mathscr{C}_{m}(\zeta), m=0,1, \ldots, n$. 
Преобразовав результат с помошью формул Коши для ограниченных и неограниченных областей с учетом

$$
\varphi_{0}(0)=0, \quad \varphi_{k}(\infty)=0, \quad k=1, \ldots, n,
$$

придем к искомой системе

$$
\begin{gathered}
M+\bar{M}+\varphi_{0}(z)+\sum_{k=1}^{n} \overline{\varphi_{k}} L_{0}(z)+\sum_{j=1}^{H} \sum_{p=1}^{N_{j}}\left(X_{p, 0}^{j}(z) B_{p}^{j}+\psi_{p, 0}^{j}(z) \overline{B_{p}^{j}}\right)=F_{0}(z) \\
\varphi_{m}(z)+\left.\sum_{\substack{k=0 \\
k \neq m}}^{n} \overline{\varphi_{k}} L_{m}(z)\right|_{\infty} ^{z}+\sum_{j=1}^{H} \sum_{p=1}^{N_{j}}\left(X_{p, m}^{j}(z) B_{p}^{j}+\psi_{p, m}^{j}(z) \overline{B_{p}^{j}}\right)=F_{m}(z), \\
m=1, \ldots, n,
\end{gathered}
$$

где

$$
\begin{aligned}
F_{m}(z) & =\frac{\delta_{m}}{\pi i} \int_{\mathscr{C}_{m}(\zeta)} \frac{f_{m}^{* *}(\zeta)}{\zeta-z} d \zeta, \quad m=0,1, \ldots, n, \\
\delta_{m} & =\left\{\begin{aligned}
1, & \text { при } m=0 \\
-1, & \text { при } m \neq 0 .
\end{aligned}\right.
\end{aligned}
$$

При этом

$$
\begin{aligned}
& X_{p, m}^{j}(z)=\frac{\delta_{m}}{2 \pi i} \int_{\mathscr{C}_{m}(\zeta)} \frac{1}{\left(\zeta-b_{j}\right)^{p}(\zeta-z)} d \zeta, \\
& \psi_{p, m}^{j}(z)=\frac{\delta_{m}}{2 \pi i} \int_{\mathscr{C}_{m}(\zeta)} \frac{1}{\left(L_{m}(\zeta)-\overline{b_{j}}\right)^{p}(\zeta-z)} d \zeta, \\
& m=0,1, \ldots, n, \quad j=1, \ldots, H, \quad p=1, \ldots, N_{j} .
\end{aligned}
$$

Полагая в (4.3) $z=0$, получаем

$$
F_{0}(0)=M+\bar{M}+\sum_{j=1}^{H} \sum_{p=1}^{N_{j}}\left(X_{p, 0}^{j}(z) B_{p}^{j}+\psi_{p, 0}^{j}(z) \overline{B_{p}^{j}}\right)
$$

Вычитая почленно из (4.3) соотношение (4.9), имеем

$$
\varphi_{0}(z)+\sum_{k=1}^{n} \overline{\varphi_{k}} L_{0}(z)=F_{0}^{\prime}(z)
$$

где

$$
F_{0}^{\prime}(z)=\left.F_{0}(z)\right|_{0} ^{z}-\left.\sum_{j=1}^{H} \sum_{p=1}^{N_{j}}\left(X_{p, 0}^{j}(z) B_{p}^{j}+\psi_{p, 0}^{j}(z) \overline{B_{p}^{j}}\right)\right|_{0} ^{z}
$$


Если ввести новые функции, определяемые формулами

$$
F_{m}^{\prime}(z)=F_{m}(z)-\left.\sum_{j=1}^{H} \sum_{p=1}^{N_{j}}\left(X_{p, m}^{j}(z) B_{p}^{j}+\psi_{p, m}^{j}(z) \overline{B_{p}^{j}}\right)\right|_{\infty} ^{z}, \quad m=1, \ldots, n
$$

то из (4.10) и (4.4) следует, что функции $\varphi_{k}(z), k=0,1, \ldots, n$, удовлетворяют системе уравнений

$$
\begin{gathered}
\varphi_{0}(z)+\left.\sum_{k=1}^{n} \overline{\varphi_{k}} L_{0}(z)\right|_{0} ^{z}=F_{0}^{\prime}(z), \\
\varphi_{m}(z)+\left.\sum_{\substack{k=0 \\
k \neq m}}^{n} \overline{\varphi_{k}} L_{m}(z)\right|_{\infty} ^{z}=F_{m}^{\prime}(z), \quad m=1, \ldots, n .
\end{gathered}
$$

Установим условия, при которых неоднородная задача Шварца имеет решение в классе однозначных аналитических функций с конечным числом полюсов различной кратности.

Отметим, что из (4.7) и (4.8) следует

$$
\overline{X_{p, m}^{j}\left(\alpha_{m}\right)}=\psi_{p, m}^{j}\left(\alpha_{m}\right), \quad m=0,1, \ldots, n, \quad j=1, \ldots, H, \quad p=1, \ldots, N_{j}
$$

Умножим обе части равенства $(4.2)$ на $\frac{1}{2 \pi i} \frac{d \zeta}{\zeta-a_{m}}, m=1, \ldots, n$, и проинтегрируем произведение по $\mathscr{C}_{m}(\zeta), m=1, \ldots, n$. Преобразовав результат с помощью формул Кошш, формул (4.7), (4.8), с учетом (4.5), (4.6), (4.12) получим

$$
\delta_{m} F_{m}^{\prime}\left(a_{m}\right)=M+\bar{M}+2 \operatorname{Re} \sum_{\substack{k=0 \\ k \neq m}}^{n} \varphi_{k}\left(a_{m}\right), \quad m=1, \ldots, n
$$

Приступая к решению системы (4.13), введем прежде всего некоторые необходимые функции и дополнительные обозначения.

Для точек $\zeta \in \mathscr{C}_{m}(\zeta), m=0,1, \ldots, n$, имеет место соотношение

$$
\left(\overline{\frac{1}{\zeta-\mathscr{A}} d \zeta}\right)=\frac{1}{\zeta-\overline{L_{m}(\mathscr{A})}} d \zeta-\frac{1}{\zeta-a_{m}} d \zeta
$$

Из (4.7) и (4.8) с учетом (4.16) получаем

$$
\begin{gathered}
\overline{\psi_{p, k}^{j}} L_{m}(z)=X_{p, k}^{j}\left(a_{k}\right)-X_{p, k}^{j} \overline{L_{k}} L_{m}(z), \\
\overline{X_{p, k}^{j}} L_{m}(z)=\psi_{p, k}^{j}\left(a_{k}\right)-\psi_{p, k}^{j} \overline{L_{k}} L_{m}(z), \quad j=1, \ldots, H, \quad p=1, \ldots, N, \\
k \neq m, \quad k, m=0,1, \ldots, n .
\end{gathered}
$$


$\mathrm{C}$ целью более компактной записи сумм функций, определенных в $\mathscr{D}_{m}$, будем использовать следующие символы:

$$
\begin{gathered}
\sum_{k_{1} \neq k_{2}}^{k_{2 \nu} \neq m}=\sum_{\substack{k_{1}=0 \\
k_{1} \neq k_{2}}}^{n} \sum_{\substack{k_{2}=0 \\
k_{2} \neq k_{3}}}^{n} \ldots \sum_{\substack{k_{2 \nu}-1=0 \\
k_{2 \nu-1} \neq k_{2 \nu}}}^{n} \sum_{\substack{k_{2 \nu}=0 \\
k_{2 \nu} \neq m}}^{n}, \\
\mathscr{D}_{2 \nu}(z)=\overline{L_{k_{1}}} L_{k_{2}} \overline{L_{k_{3}}} \cdots \overline{L_{k_{2 \nu}-1}} L_{k_{2 \nu}}(z) \\
T_{k, \nu, m}(z)=L_{k} \overline{\mathscr{D}_{2 \nu}} L_{m}(z) .
\end{gathered}
$$

Пусть $\widehat{b_{0}}=0, \widehat{b_{m}}=\infty, m=1, \ldots, n$. Тогда система (4.13) записьвается более компактно

$$
\varphi_{m}(z)+\left.\sum_{\substack{k=0 \\ k \neq m}}^{n} \overline{\varphi_{k}} L_{m}(z)\right|_{\widehat{b_{m}}} ^{z}=F_{m}^{\prime}(z), \quad m=0,1, \ldots, n
$$

Систему (4.21) можно преобразовать в систему линейных функциональных уравнений, не содержащих сопряженных функций. С этой целью в (4.21) заменим $k$ на $k_{1}$ и $m$ на $k$. Подставив полученное значение $\varphi_{k}(z)$ во второе слагаемое левой части формулы (4.21), находим

$$
\varphi_{m}(z)-\left.\sum_{\substack{k_{1}=0 \\ k_{1} \neq k}}^{n} \sum_{\substack{k=0 \\ k \neq m}}^{n} \varphi_{k_{1}} \overline{L_{k}} L_{m}(z)\right|_{\widehat{b_{m}}} ^{z}=E_{m}(z), \quad m=0,1, \ldots, n
$$

где

$$
E_{m}(z)=F_{m}^{\prime}(z)-\left.\sum_{\substack{k=0 \\ k \neq m}}^{n} \overline{F_{k}^{\prime}} L_{m}(z)\right|_{\widehat{b_{m}}} ^{z}
$$

Из (4.23) и (4.12) с учетом $(4.17),(4.18)$ следует

$$
\begin{aligned}
E_{m}(z)=\sum_{k=0}^{n}\left(F_{k} \overline{L_{k}} L_{m}(z)\right. & \\
& \left.-\sum_{j=1}^{H} \sum_{p=1}^{N_{j}}\left(X_{p, k}^{j} \overline{L_{k}} L_{m}(z) B_{p}^{j}+\psi_{p, k}^{j} \overline{L_{k}} L_{m}(z) \overline{B_{p}^{j}}\right)\right)\left.\right|_{\widehat{b_{m}}} ^{z} .
\end{aligned}
$$

Посредством вычислений, аналогичных использованным в работе [1] (см. [1, 2 , вьвод системы уравнений (2.23)]), получаем

$$
\varphi_{m}(z)=\left.\sum_{\nu=1}^{\infty} \sum_{k \neq k_{1}}^{k_{2 \nu} \neq m} E_{k} \mathscr{D}_{2 \nu-1} L_{m}(z)\right|_{\widehat{b_{m}}} ^{z}+E_{m}(z), \quad m=0,1, \ldots, n
$$


Из (4.25) и (4.24) с учетом (4.20), (4.5) находим решение системы (4.21) линейных функциональных уравнений

$$
\begin{array}{r}
\varphi_{m}(z)=\sum_{k=0}^{n} \sum_{\nu=0}^{\infty} \sum_{k_{1} \neq k_{2}}^{k_{2 \nu} \neq m}\left(F_{k} T_{k, \nu, m}(z)-\sum_{j=1}^{H} \sum_{p=1}^{N_{j}}\left(X_{p, k}^{j} T_{k, \nu, m}(z) B_{p}^{j}\right.\right. \\
\left.\left.+\psi_{p, k}^{j} T_{k, \nu, m}(z) \overline{B_{p}^{j}}\right)\right)\left.\right|_{\widehat{b_{m}}} ^{z}, \quad m=0,1, \ldots, n .
\end{array}
$$

Подставив значение $\varphi_{m}(z)$ из (4.26) в правую часть соотношений $(4.15)$, а значение $F_{m}^{\prime}(z)$ из (4.12) в левую часть (4.15), получаем систему линейных алгебраических уравнений относительно $B_{p}^{j}$ и $\overline{B_{p}^{j}}$

$$
\sum_{j=1}^{H} \sum_{p=1}^{N_{j}}\left(G_{p, m}^{j} B_{p}^{j}+\overline{G_{p, m}^{j}} \overline{B_{p}^{j}}\right)=F_{m}^{\prime \prime}, \quad m=1, \ldots, n
$$

где

$$
\begin{aligned}
G_{p, m}^{j}= & X_{p, m}^{j}\left(a_{m}\right)+X_{p, 0}^{j}(0) \\
& +\left.\sum_{\kappa=0}^{n} \sum_{\nu=0}^{\infty} \sum_{k_{1} \neq k_{2}}^{k_{2 \nu} \neq k} \sum_{\substack{k=0 \\
k \neq m}}^{n}\left(X_{p, \kappa}^{j} T_{\kappa, \nu, k}(z)-X_{p, \kappa}^{j} \overline{L_{\kappa} T_{\kappa, \nu, k}}(z)\right)\right|_{\widehat{b_{k}}} ^{a_{m}} \\
F_{m}^{\prime \prime}= & F_{m}\left(a_{m}\right)+F_{0}(0) \\
& +\left.\sum_{\kappa=0}^{n} \sum_{\nu=0}^{\infty} \sum_{k_{1} \neq k_{2}}^{k_{2 \nu} \neq k} \sum_{\substack{k=0 \\
k \neq m}}^{n}\left(F_{\kappa} T_{\kappa, \nu, k}(z)-F_{\kappa} \overline{L_{\kappa} T_{\kappa, \nu, k}(z)}\right)\right|_{\widehat{b_{k}}} ^{a_{m}}
\end{aligned}
$$

Система (4.27) имеет $n$ уравнений с

$$
\widetilde{N}=2 \sum_{j=1}^{H} N_{j}
$$

неизвестными.

Пусть $r$ - ранг системы (4.27). Будем считать, что первые $r$ уравнений системы (4.27) образуют базис. Произведем перенумерацию неизвестных. Вместо $B_{p}^{j}$ и $\overline{B_{p}^{j}}$ обозначим неизвестные $\widehat{B_{p}^{j}}$. Если $\widehat{B_{p}^{j}}$ - базисное неизвестное, то будем считать, что индексы $j, p \in \mathscr{P}_{\delta}$. Если же $\widehat{B_{p}^{j}}-$ свободное неизвестное, то $j, p \in \mathscr{P}_{c}$. Тогда (4.27) принимает вид:

$$
\sum_{j, p \in \mathscr{P}_{\delta}} \widehat{G_{p, m}^{j}} \widehat{B_{p}^{j}}=F_{m}^{\prime \prime}-\sum_{j, p \in \mathscr{P}_{c}} \widehat{G_{p, m}^{j}} \widehat{B_{p}^{j}}, \quad m=1, \ldots, r
$$


Пусть $\Delta$ - основной определитель $(4.31), \Delta \neq 0,{\widehat{\left(G_{p, m}^{j}\right.}}^{*}$ есть алгебраическое дополнение элемента $\widehat{G_{p, m}^{j}}$ в определителе $\Delta$. Из (4.31) по формулам Крамера имеем

$$
\widehat{B_{p}^{j}}=\sum_{m=1}^{r} \frac{{\widehat{\left(G_{p, m}^{j}\right)}}^{*}}{\Delta}\left(F_{m}^{\prime \prime}-\sum_{j, p \in \mathscr{P}_{c}} \widehat{G_{p, m}^{j}} \widehat{B_{p}^{j}}\right), \quad j, p \in \mathscr{P}_{\delta} .
$$

Если $r<n$, то правые части (4.27) подчинены $(n-r)$ условиям совместности системы $[10$, гл. $2, \S 1]$

$$
F_{m}^{\prime}=\sum_{k=1}^{r} \lambda_{k}^{m} F_{k}^{\prime \prime}, \quad m=r+1, \ldots, n
$$

где $\lambda_{k}^{m}$ - определяются с помощью формул (4.28). Из (4.29), (4.5) следует, что (4.33) - условия согласованности граничных значений $f_{m}^{* *}(\zeta)$ вешественной части искомой функции $f^{* *}(z)$ :

$$
\begin{aligned}
\frac{\delta_{m}}{\pi i} \int_{\mathscr{C}_{m}(\zeta)} & \frac{f_{m}^{* *}(\zeta)}{\zeta-a_{m}} d \zeta \\
= & \frac{1}{\pi i}\left(\sum_{k=1}^{r} \lambda_{k}^{m}-1\right) \int_{\mathscr{C}_{0}(\zeta)} \frac{f_{0}^{* *}(\zeta)}{\zeta} d \zeta+\sum_{k=1}^{r} \lambda_{k}^{m} \frac{\delta_{k}}{\pi i} \int_{\mathscr{C}_{k}(\zeta)} \frac{f_{k}^{* *}(\zeta)}{\zeta-a_{k}} d \zeta \\
& \quad+\sum_{\kappa=0}^{n} \frac{\delta_{\kappa}}{\pi i} \int_{\mathscr{C}_{\kappa}(\zeta)} f_{\kappa}^{* *}(\zeta) \mathscr{K}_{\kappa, m}^{\prime}(\zeta) d \zeta, \quad m=r+1, \ldots, n,
\end{aligned}
$$

где

$$
\begin{aligned}
\mathscr{K}_{\kappa, m}^{\prime}(\zeta) & =\sum_{k=1}^{r} \lambda_{k}^{m} \mathscr{K}_{\kappa, k}(\zeta)-\mathscr{K}_{\kappa, m}(\zeta), \\
\mathscr{K}_{\kappa, m}(\zeta) & =\left.\sum_{\nu=0}^{\infty} \sum_{k_{1} \neq k_{2}}^{k_{2 \nu} \neq k} \sum_{\substack{k=0 \\
k \neq m}}^{n}\left(\frac{1}{\zeta-T_{\kappa, \nu, k}(z)}-\frac{1}{\zeta-\overline{L_{\kappa} T_{\kappa, \nu, k}(z)}}\right)\right|_{\widehat{b_{k}}} ^{a_{m}} .
\end{aligned}
$$

Пусть $\mathscr{P}_{\delta}=\mathscr{P}_{\delta}^{\prime}+\mathscr{P}_{\delta}^{\prime \prime}, \mathscr{P}_{c}=\mathscr{P}_{c}^{\prime}+\mathscr{P}_{c}^{\prime \prime}$, где $\mathscr{P}_{\delta}-$ множество индексов $(j, p)$, соответствуюших базисным неизвестным $B_{p}^{j}, \mathscr{P}_{\delta}^{\prime \prime}$ - множество индексов $(j, p)$, соответствуюших базисным неизвестным $\overline{B_{p}^{j}}, \mathscr{P}_{c}^{\prime}-$ множество индексов $\left(j_{0}, p_{0}\right)$, соответствуюших свободным неизвестным $B_{p_{0}}^{j_{0}}, \mathscr{P}_{c}^{\prime \prime}-$ множество индексов $\left(j_{0}, p_{0}\right)$, соответствуюших свободным неизвестным $\frac{B_{p_{0}}^{j_{0}}}{\text {. Из }}$ И..32) следует

$$
\begin{aligned}
& B_{p}^{j}=\sum_{m=1}^{r} \frac{\left(G_{p, m}^{j}\right)^{*}}{\Delta}\left(F_{m}^{\prime \prime}-\sum_{j, p \in \mathscr{P}_{c}^{\prime}} G_{p_{0}, m}^{j_{0}} B_{p_{0}}^{j_{0}}-\sum_{j, p \in \mathscr{P}_{c}^{\prime \prime}} \overline{G_{p_{0}, m}^{j_{0}}} \overline{B_{p_{0}}^{j_{0}}}\right), \quad j, p \in \mathscr{P}_{\delta}^{\prime}, \\
& \overline{B_{p}^{j}}=\sum_{m=1}^{r} \frac{\overline{\left(G_{p, m}^{j}\right)^{*}}}{\Delta}\left(F_{m}^{\prime \prime}-\sum_{j, p \in \mathscr{P}_{c}^{\prime}} G_{p_{0}, m}^{j_{0}} B_{p_{0}}^{j_{0}}-\sum_{j, p \in \mathscr{P}_{c}^{\prime \prime}} \overline{G_{p_{0}, m}^{j_{0}}} \overline{B_{p_{0}}^{j_{0}}}\right), \quad j, p \in \mathscr{P}_{\delta}^{\prime \prime} .
\end{aligned}
$$


Подставив в формулу (4.9) соотношения (4.37), (4.38), получаем

$$
\begin{aligned}
M+\bar{M}=F_{0}(0)-\sum_{m=1}^{r} G_{m} F_{m}^{\prime \prime} & +\sum_{j, p \in \mathscr{P}_{c}^{\prime}}\left(X_{p_{0}, 0}^{j_{0}}(0)+\sum_{m=1}^{r} G_{p_{0}, m}^{j_{0}} G_{m}\right) B_{p_{0}}^{j_{0}} \\
& +\sum_{j, p \in \mathscr{P}_{c}^{\prime \prime}}\left(\psi_{p_{0}, 0}^{j_{0}}(0)+\sum_{m=1}^{r} \overline{G_{p_{0}, m}^{j_{0}}} G_{m}\right) \overline{B_{p_{0}}^{j_{0}}}
\end{aligned}
$$

где

$$
G_{m}=\frac{1}{\Delta} \sum_{j, p \in \mathscr{P}_{\delta}^{\prime}} X_{p_{0}, 0}^{j_{0}}(0)\left(G_{p, m}^{j}\right)^{*}+\sum_{j, p \in \mathscr{P}_{\delta}^{\prime \prime}} \psi_{p_{0}, 0}^{j_{0}}(0)\left(\overline{G_{p, m}^{j}}\right)^{*}, \quad m=1, \ldots, r
$$

Используя разбиение множества индексов, правую часть формулы (4.1) можно преобразовать к виду

$$
\begin{aligned}
f^{* *}(z)=\operatorname{Re} M+ & i \operatorname{Im} M \\
+ & \sum_{k=0}^{n} \varphi_{k}(z)+\sum_{j, p \in \mathscr{P}_{\delta}^{\prime}} \frac{B_{p}^{j}}{\left(z-b_{j}\right)^{p}}+\sum_{j_{0}, p_{0} \in \mathscr{P}_{c}^{\prime}} \frac{B_{p_{0}}^{j_{0}}}{\left(z-b_{j, 0}\right)^{p_{0}}} .
\end{aligned}
$$

Подставив в (4.41) соотношения (4.36)-(4.39), с учетом $(4.29),(4.5)$ получаем

$$
\begin{aligned}
f^{* *}(z)=\sum_{\kappa=0}^{n} \frac{\delta_{\kappa}}{\pi i} \int_{\mathscr{C}_{\kappa}(\zeta)} f_{\kappa}^{* *}(\zeta) H_{\kappa}(z, \zeta) d \zeta+i \operatorname{Im} M \\
+\sum_{j_{0}, p_{0} \in \mathscr{P}_{c}^{\prime}} X\left(z, j_{0}, p_{0}\right) B_{p_{0}}^{j_{0}}+\sum_{j_{0}, p_{0} \in \mathscr{P}_{c}^{\prime \prime}} \psi\left(z ; j_{0}, p_{0}\right) \overline{B_{p_{0}}^{j_{0}}}
\end{aligned}
$$

где

$$
H_{\kappa}(z, \zeta)= \begin{cases}H_{0, r}^{\prime}(z, \zeta)+\frac{1}{\zeta} \sum_{m=1}^{r} H_{m}(z)+\frac{1}{2 \zeta}, & \text { если } \kappa=0, \\ H_{\kappa, r}^{\prime}(z, \zeta)+\frac{H_{\kappa}(z)}{\zeta-a_{\kappa}}, & \text { если } 1 \leqslant \kappa \leqslant r, \\ H_{\kappa, r}^{\prime}(z, \zeta), & \text { если } r<\kappa \leqslant n .\end{cases}
$$

При этом

$$
\begin{aligned}
& H_{\kappa, r}^{\prime}(z, \zeta)= \sum_{\nu=0}^{\infty} \sum_{k_{1} \neq k_{2}}^{k_{2 \nu} \neq k}\left(\left.\sum_{k=0}^{n} \frac{1}{\zeta-T_{\kappa, \nu, k}(z)}\right|_{\widehat{b_{k}}} ^{z}\right. \\
&\left.+\sum_{\substack{k=0 \\
k \neq m}}^{n} \sum_{m=1}^{r} H_{m}(z)\left(\left.\frac{1}{\zeta-T_{\kappa, \nu, k}(z)}\right|_{\widehat{b_{k}}} ^{a_{m}}-\left.\frac{1}{\zeta-\overline{L_{\kappa} T_{\kappa, \nu, k}(z)}}\right|_{\widehat{b_{k}}} ^{a_{m}}\right)\right), \\
& \kappa=0,1, \ldots, n .
\end{aligned}
$$


Здесь

$$
\begin{gathered}
H_{m}(z)=\sum_{j, p \in \mathscr{P}_{\delta}^{\prime}} \frac{\left(G_{p, m}^{j}\right)^{*}}{\Delta\left(z-b_{j}\right)^{p}}-\frac{1}{2} G_{m}-\sum_{\kappa=0}^{n} \sum_{\nu=0}^{\infty} \sum_{k_{1} \neq k_{2}}^{k_{2 \nu} \neq k} \sum_{k=0}^{n} \frac{1}{\Delta} \\
\times\left.\left(\sum_{j, p \in \mathscr{P}_{\delta}^{\prime}} X_{p, \kappa}^{j} T_{\kappa, \nu, k}(z)\left(G_{p, m}^{j}\right)^{*}+\sum_{j, p \in \mathscr{P}_{\delta}^{\prime \prime}} \psi_{p, \kappa}^{j} T_{\kappa, \nu, k}(z) \overline{\left(G_{p, m}^{j}\right)^{*}}\right)\right|_{\widehat{b_{k}}} ^{z}, \\
m=1, \ldots, r .
\end{gathered}
$$

Кроме того,

$$
\begin{aligned}
X\left(z ; j_{0}, p_{0}\right)= & \left(z-b_{j, 0}\right)^{-p_{0}}+\frac{1}{2} X_{p_{0}, 0}^{j_{0}}(0)-\sum_{m=1}^{r} G_{p_{0}, m}^{j_{0}} H_{m}(z) \\
& -\left.\sum_{\kappa=0}^{n} \sum_{\nu=0}^{\infty} \sum_{k_{1} \neq k_{2}}^{k_{2 \nu} \neq k} \sum_{k=0}^{n} X_{p_{0}, \kappa}^{j_{0}} T_{\kappa, \nu, k}(z)\right|_{\widehat{b_{k}}} ^{z}, \quad\left(j_{0}, p_{0}\right) \in \mathscr{P}_{c}^{\prime}, \\
\psi\left(z ; j_{0}, p_{0}\right)= & \frac{1}{2} \psi_{p_{0}, 0}^{j_{0}}(0)-\sum_{m=1}^{r} \overline{G_{p_{0}, m}^{j_{0}}} H_{m}(z) \\
& -\left.\sum_{\kappa=0}^{n} \sum_{\nu=0}^{\infty} \sum_{k_{1} \neq k_{2}}^{k_{2 \nu} \neq k} \sum_{k=0}^{n} \psi_{p_{0}, \kappa}^{j_{0}} T_{\kappa, \nu, k}(z)\right|_{\widehat{b_{k}}} ^{z}, \quad\left(j_{0}, p_{0}\right) \in \mathscr{P}_{c}^{\prime \prime} .
\end{aligned}
$$

Доказательство равномерной сходимости на $\mathscr{E}$, т.е. внутри $\mathscr{K} \backslash\left(\bigcup_{j} b_{j}\right)$, рядов интегрального представления (4.42) осушествляется по схеме, предложенной в $[1, \S 4]$.

Задаваемая формулой (4.42) функция $f^{* *}(z)$ при выполнении условий согласованности граничных значений (4.34) регулярна и однозначна в $\mathscr{K} \backslash\left(\bigcup_{j} b_{j}\right)$. Поступая так же, как и в $[1, \S 4]$, докажем, что вещественная часть функции $f^{* *}(z)$, задаваемой формулой $(4.42)$, совпадает на $\mathscr{C}_{m}(\zeta)$ с $f_{m}^{* *}(\zeta), m=0,1, \ldots, n$.

Итак, приходим к теореме.

Tеорема 4.1. Пусть функиия $f^{* *}(z)$ аналитическая в конечносвязной круговой области $\mathscr{K}$, кроме заданных точек $b_{j}, j=1, \ldots, H$, әде функиия $f^{* *}(z)$ имеет кратные полюсь, кратностей, соответственно, $N_{j}, j=1, \ldots, H$. Пусть вещественная часть функиии $f^{* *}(z)$ на граничных компонентах $\mathscr{C}_{k}(\zeta)$ принимает непрерывные значения $f_{k}^{* *}(\zeta)$. Пусть $r$ - ранг системы (4.27), $r \leqslant \min \{n, \widetilde{N}\}$.

Возможны три случая.

1) Если $r=n \leqslant \widetilde{N}$, где $\widetilde{N}$ определяется формулой (4.30), тогда задача Швариа для мероморфных функиий в $(n+1)$-связной круговой области безусловно разрешима и решение, зависящее от $\widetilde{N}-n+1$ произвольных вещественных постоянных, определяется формулами (4.42)-(4.47) с учетом (4.40), (4.28).

2) Eсли $r<\min \{n, \widetilde{N}\}$, то задача Шварца для мероморфных функций в $(n+1)$-связной круговой области разрешима при выполнении $(n-r)$ условий 
согласованности граничных значений функций $f_{k}^{* *}(\zeta), k=0,1, \ldots, n$, определяемых соотношениями (4.34). Решение задачи, зависящее от $\widetilde{N}-r+1$ произвольных вещественных постоянных, определяется формулами (4.42)-(4.47).

3) Если $r=\widetilde{N}<n$, то задача Шварца для мероморфных функций в $(n+1)$ связной круговой области разрешима, когда $f_{k}^{* *}(\zeta), k=0,1, \ldots, n$, удовлетворяют $(n-r)$ условиям (4.34), и имеет место формула

$$
f^{* *}(z)=\sum_{\kappa=0}^{n} \frac{\delta_{\kappa}}{\pi i} \int_{\mathscr{C}_{\kappa}(\zeta)} f_{\kappa}^{* *}(\zeta) H_{\kappa}(z, \zeta) d \zeta+i \mathscr{D}
$$

где $\mathscr{D}$ - произвольное вещественное число, $H_{\kappa}(z, \zeta)$ определяется формулами (4.43)-(4.47) с учетом (4.40), (4.28).

СлЕДСТВИЕ 4.1.1. Если в формуле (4.48) положсить $\widetilde{N}=0$, то получаем формулу Шварца для конечносвязных круговых областей [1].

СлЕДСТВИЕ 4.1.2. Если в условиях предыдущего следствия 4.1.1 положсть $n=1$, то получаем известную формулу Вилля [4].

СлЕДСТВИЕ 4.1.3. Если в условиях следствия 4.1 .2 устремить $R_{1}$ к нулю, то в результате предельного перехода получаем формулу Швариа [9].

\section{§5. Многолистная формула \\ Тири-Ахиезера-Голузина в $\mathscr{K}\left(R_{j}, a_{j}, j=0, \ldots, n\right)$}

ТЕОРема 5.1. Пусть функиия $f^{* *}(z)$ аналитическая в конечносвязной круговой области $\mathscr{K}$, кроме заданных точек $b_{j}, j=1, \ldots, H$, әде функиия $f^{* *}(z)$ имеет кратные полюсы, кратностей, соответственно, $N_{j}, j=1, \ldots, H$. Пусть $r$ - ранг системь (4.27), $r \leqslant \min \{n, \tilde{N}\}$.

Возможсни три случая.

1) Если $r=n \leqslant \tilde{N}$, где $\widetilde{N}$ определяется формулой (4.30), тогда структурная формула

$$
f^{* *}(z)=C z^{\mathscr{A}_{0}+\beta+i \mathscr{D}^{\prime}} Q(z) \exp \left(\frac{1}{2 \pi} S(z)\right),
$$

зависящая от $\widetilde{N}-n+1$ произвольных вещественных постоянных

$$
\operatorname{Re} B_{p}^{j} \quad n p u \quad j, p \in \mathscr{P}_{c}^{\prime} \quad u \quad \operatorname{Im} B_{p}^{j} \quad n p u \quad j, p \in \mathscr{P}_{c}^{\prime \prime},
$$

является необходимым и достаточным условием принадлехсности функиии $f^{* *}(z)$ классу $\mathscr{B}_{N}^{*}\left(R_{j}, a_{j}, j=0, \ldots, n\right)$ и определяется формулами (4.42)-(4.47) с учетом (4.40), (4.28). Причем

$$
Q(z)=\prod_{j, p \in \mathscr{P}_{c}^{\prime}}\left(\exp \left(B_{p_{0}}^{j_{0}} X\left(z ; j_{0}, p_{0}\right)\right)\right) \prod_{j, p \in \mathscr{P}_{c}^{\prime \prime}}\left(\exp \left(\overline{B_{p_{0}}^{j_{0}}} \psi\left(z ; j_{0}, p_{0}\right)\right)\right)
$$


Кроме того,

$$
\begin{aligned}
S(z)=\sum_{\kappa=0}^{n} \delta_{\kappa} \int_{-\pi}^{\pi} \sum_{\nu=0}^{\infty} \sum_{k_{1} \neq k_{2}}^{k_{2 \nu} \neq k} \sum_{k=0}^{n} B_{\kappa, \nu, k}\left(a_{k}+R_{k} \exp (i \vartheta)\right) \\
\times \ln \left(z-M_{k, \nu, \kappa}\left(a_{k}+R_{k} \exp (i \vartheta)\right)\right) d \mu_{\kappa}(\vartheta) .
\end{aligned}
$$

2) Eсли $r<\min \{n, \widetilde{N}\}$, то при выполнении $(n-r)$ условий

$$
\begin{aligned}
\delta_{m} \mathscr{A}_{m} & =\left(\sum_{k=1}^{r} \lambda_{k}^{m}-1\right) \mathscr{A}_{0}+\sum_{k=1}^{r} \lambda_{k}^{m} \delta_{k} \mathscr{A}_{k} \\
& +\sum_{\kappa=0}^{n} \frac{\delta_{\kappa}}{\pi} \int_{-\pi}^{\pi} \mathscr{K}_{\kappa, m}^{\prime}\left(a_{\kappa}+R_{\kappa} \exp (i \vartheta)\right) d \mu_{\kappa}(\vartheta), \quad m=r+1, \ldots, n,
\end{aligned}
$$

структурная формула, зависящая от $\widetilde{N}-r+1$ произвольньх вещественнъх постоянных (5.2), является необходимым и достаточным условием принадлежности функции $f^{* *}(z)$ классу $\mathscr{B}_{N}^{*}\left(R_{j}, a_{j}, j=0, \ldots, n\right)$ и определяется формулой (5.1).

3) Если $r=\widetilde{N}<n$, то при выполнении $(n-r)$ условий (5.5) структурная формула

$$
f^{* *}(z)=C z^{\mathscr{A}_{0}+\beta+i \mathscr{D}} Q(z) \exp \left(\frac{1}{2 \pi} S(z)\right)
$$

является необходимым и достаточным условием принадлежсности функиии $f^{* *}(z)$ классу $\mathscr{B}_{N}^{*}\left(R_{j}, a_{j}, j=0, \ldots, n\right)$. Здесь $S(z)$ определяется формулой (5.4), $\mathscr{D}$ - произвольное вещественное число. Причем

$$
Q(z)=\prod_{\nu=0}^{\infty} \prod_{k_{1} \neq k_{2}}^{k_{2 \nu} \neq k} \prod_{k=0}^{n}\left(\frac{G_{m, \nu, k}\left(z, N_{0}\right)}{G_{m, \nu, k}\left(z, N_{p}\right)}\right) .
$$

Доказательство теоремы 5.1 проводится аналогично доказательству теоремы 3.2 с учетом интегральных представлений (4.42), (4.48).

СЛЕДСТВИЕ 5.1.1. Если в условиях теоремы 5.1 положить $R_{0}=1, n=1$, то получаем известную теорему Зморовича [5].

СЛЕДСТВИЕ 5.1.2. Если в условиях теоремы 5.1 в случае 3) предположить, что $R_{k}, k=1, \ldots, n$, достаточно мальи, а $R_{0}$ достаточно велико, то получаем известную теорему Максимова [8].

Теорема 5.2. Пусть функиия $f^{* *}(z)$ аналитическая в конечносвязной круговой области $\mathscr{K}$, кроме заданных точек $b_{j}, j=1, \ldots, H$, где функиия $f^{* *}(z)$ имеет кратные полюсы, кратностей, соответственно, $N_{j}, j=1, \ldots, H$. Пусть $r-$ ранг системь $(4.27), r \leqslant \min \{n, \tilde{N}\}$.

Возможны три случая. 
1) Если $r=n \leqslant \tilde{N}$, где $\widetilde{N}$ определяется формулой (4.30), тогда структурная формула

$$
f^{* *}(z)=C \int z^{\mathscr{A}_{0}-1+\beta+i \mathscr{D}^{\prime}} Q(z) \exp \left(\frac{1}{2 \pi} S(z)\right) d z+C_{1},
$$

зависящая от $\widetilde{N}-n+1$ произвольных вещественных постоянных

$$
\operatorname{Re} B_{p}^{j} \quad n p u \quad j, p \in \mathscr{P}_{c}^{\prime} \quad u \quad \operatorname{Im} B_{p}^{j} \quad n p u \quad j, p \in \mathscr{P}_{c}^{\prime \prime},
$$

является необходимым и достаточным условием принадлежсности функиии $f^{* *}(z)$ классу $\mathscr{B}_{N}^{0}\left(R_{j}, a_{j}, j=0, \ldots, n\right)$ и определяется формулами (4.42)-(4.47) с учетом (4.40), (4.28). Причем

$$
Q(z)=\prod_{j, p \in \mathscr{P}_{c}^{\prime}}\left(\exp \left(B_{p_{0}}^{j_{0}} X\left(z ; j_{0}, p_{0}\right)\right)\right) \prod_{j, p \in \mathscr{P}_{c}^{\prime \prime}}\left(\exp \left(\overline{B_{p_{0}}^{j_{0}}} \psi\left(z ; j_{0}, p_{0}\right)\right)\right)
$$

Кроме того,

$$
\begin{aligned}
S(z)=\sum_{\kappa=0}^{n} \delta_{\kappa} \int_{-\pi}^{\pi} \sum_{\nu=0}^{\infty} \sum_{k_{1} \neq k_{2}}^{k_{2 \nu} \neq k} \sum_{k=0}^{n} B_{\kappa, \nu, k}\left(a_{k}+R_{k} \exp (i \vartheta)\right) \\
\times \ln \left(z-M_{k, \nu, \kappa}\left(a_{k}+R_{k} \exp (i \vartheta)\right)\right) d \mu_{\kappa}(\vartheta) .
\end{aligned}
$$

2) Если $r<\min \{n, \tilde{N}\}$, то при выполнении $(n-r)$ условий

$$
\begin{aligned}
\delta_{m} \mathscr{A}_{m} & =\left(\sum_{k=1}^{r} \lambda_{k}^{m}-1\right) \mathscr{A}_{0}+\sum_{k=1}^{r} \lambda_{k}^{m} \delta_{k} \mathscr{A}_{k} \\
+ & \sum_{\kappa=0}^{n} \frac{\delta_{\kappa}}{\pi} \int_{-\pi}^{\pi} \mathscr{K}_{\kappa, m}^{\prime}\left(a_{\kappa}+R_{\kappa} \exp (i \vartheta)\right) d \mu_{\kappa}(\vartheta), \quad m=r+1, \ldots, n,
\end{aligned}
$$

структурная формула, зависящая от $\widetilde{N}-r+1$ произвольных вещественных постоянных (5.9), является необходимым и достаточным условием принадлежнности функиии $f^{* *}(z)$ классу $\mathscr{B}_{N}^{0}\left(R_{j}, a_{j}, j=0, \ldots, n\right)$ и определяется формулой (5.8).

3) Если $r=\widetilde{N}<n$, то при выполнении $(n-r)$ условий (5.12) структурная формула

$$
f^{* *}(z)=C \int z^{\mathscr{A}_{0}-1+\beta+i \mathscr{D}} Q(z) \exp \left(\frac{1}{2 \pi} S(z)\right) d z+C_{1}
$$

является необходимым и достаточным условием принадлежкности функчии $f^{* *}(z)$ классу $\mathscr{B}_{N}^{0}\left(R_{j}, a_{j}, j=0, \ldots, n\right)$. Здесь $S(z)$ определяется формулой (5.11), D - произвольное вещественное число. Причем

$$
Q(z)=\prod_{\nu=0}^{\infty} \prod_{k_{1} \neq k_{2}}^{k_{2 \nu} \neq k} \prod_{k=0}^{n}\left(\frac{G_{m, \nu, k}\left(z, N_{0}\right)}{G_{m, \nu, k}\left(z, N_{p}\right)}\right) .
$$

Доказательство теоремы 5.2 проводится аналогично доказательству теоремы 3.3 с учетом интегральных представлений (4.42), (4.48). 
СлЕДСТВИЕ 5.2.1. Если в условиях теоремы 5.2 положить $R_{0}=1, n=1$, то получаем известную теорему Зморовича [5].

СлЕДСТВИЕ 5.2.2. Если в условиях теоремь 5.2 в случае 3) предположить, что $R_{k}, k=1, \ldots, n$, достаточно маль, а $R_{0}$ достаточно велико, то получаем известную теорему Максимова [8].

СледСтвИЕ 5.2.3. Если в условиях теоремъ 5.2 предположить, что функиии $\mu_{k}(\vartheta)$, входящие в формулу (5.13), ступенчатые с точками разрьва $\vartheta_{k, j_{k}}$, $k=0, \ldots, n, j_{k}=1, \ldots, \nu_{k},-\pi<\vartheta_{k_{1}}<\cdots<\vartheta_{k_{\nu_{k}}}<\pi$ и скачками в этих точках $2 \pi \sigma_{k, j_{k}}\left(\sigma_{k, j_{k}}>0, \sum_{j_{k}=1}^{\nu_{k}} \sigma_{k, j_{k}}=1\right)$, то формула (5.13) дает регулярньее, однолистнье в $\mathscr{K}\left(R_{j}, a_{j}, j=0, \ldots, n\right)$ функции $f^{* *}(z)$, отображающие $\mathscr{K}\left(R_{j}, a_{j}, j=0, \ldots, n\right)$ на области, ограниченные выпуклыми многоугольниками с вериинами $f^{* *}\left(a_{k}+R_{k} \exp \left(i \theta_{k, j_{k}}\right)\right)$ и внутренними углами при них, равными $\pi\left(1-2 \sigma_{k, j_{k}}\right)$.

СлЕДСТВИЕ 5.2.4. Если в условиях предъдуущего следствия 5.2.3 положить $n=1$, то получаем известную теорему Тири-Ахиезера-Голузина (см. [9, гл. $7, \S 4$, п. 104, пп. 9]).

\section{Список литературы}

1. Александров И. А., Сорокин А. С. Задача Шварца для многосвязных круговых областей // Сиб. матем. журн. 1972. Т. 13. № 5. С. 971-1001.

2. Александров И.А., Сорокин А. С. О распространении вариационного метода Голузина-Куфарева на многосвязные области // Докл. АН СССР. 1967. Т. 175. №6. C. $1207-1210$.

3. Сорокин A. С. Задача Келдьша-Седова для круговых многосвязных областей // Сиб. матем. журн. 1995. Т. 36. № 1. С. 186-202.

4. Зморович B. A. О некоторых классах аналитических функций, однолистных в круговом кольце // Матем. сб. 1953. Т. 32 (74). №3. С. 633-652.

5. Зморович $B$. A. О некоторых классах аналитических функций, однолистных в круговом кольце // Матем. сб. 1956. Т. 40 (82). №2. С. 225-238.

6. Paatero $V$. Über die konforme Abbildung mehrblättriger Gebiete von beschränkter Randdrehung // Ann. Acad. Sci. Fenn. Ser. A I. 1952. V. 128. № 2. P. 23-29.

7. Paatero $V$. Über die konformen Abbildungen von Gebieten, deren Ränder von beschränkter Drehung sind // Ann. Acad. Sci. Fenn. Ser. A. 1931. V. 33. №9. P. 1-79.

8. Максимов Ю. Д. Обобшение структурной формулы для выпуклых однолистных функций на случай многосвязной круговой области // Докл. АН СССР. 961. Т. 136. № 2 . C. $284-287$.

9. Лаврентьев М.А., Шабат Б. В. Методы теории функций комплексного переменного. М.-Л.: Физматгиз, 1973.

10. Гантмахер Ф. Р. Теория матриц. М.: Наука, 1953.

Сибирская государственная горно-металлургическая академия, Новокузнецк
Поступила в редакцию 24.12 .1996 